\title{
Instrumentos da prática clínica com versão em português e a abrangência de seus conteúdos usando a CIF como referência: uma revisão sistemática
}

\author{
Health assessment instruments in Portuguese version and its comprehensiveness with \\ ICF framework: a systematic review
}

Instrumentos de la práctica clinica con versión en portugués y alcance de sus contenidos tomando como referencia la CIF: una revisión sistemática

\begin{abstract}
Karoleen Oswald Scharan', Rafaella Stradiotto Bernardelli², Katren Pedroso Corrêa ${ }^{3}$, Auristela Duarte de Lima Moser ${ }^{4}$
\end{abstract}

RESUMO I A prática clínica tem sido subsidiada por instrumentos que permitem acessar e registrar informações de funcionalidade e saúde dos indivíduos. Uma forma de conhecer qual conteúdo da Classificação Internacional de Funcionalidade, Incapacidade e Saúde (CIF) os instrumentos contemplam é usá-la como referência para auxiliar o profissional na escolha do mais adequado para acessar informações biopsicossociais. O objetivo foi identificar instrumentos da prática clínica em saúde que tiveram seu conteúdo ligado com a CIF e a existência de versão na língua portuguesa deles. Para tanto foi realizada uma revisão sistemática nas bases de dados SciELO Brasil, Lilacs e PubMed com os descritores "CIF", "questionário" e "regra de ligação" em português e inglês. Três pesquisadores independentes realizaram a seleção, e o nível de concordância foi obtido pelo coeficiente Kappa. Os critérios de elegibilidade foram: estudos primários de ligação de conteúdo de questionários, escalas, índices e checklists com a CIF publicados após 2001 em língua portuguesa ou inglesa. Foram incluídos 61 artigos, sendo 19 de origem brasileira. Dos 250 instrumentos ligados à CIF, 158 (63,2\%) apresentaram versão em português sendo que dos 37 que mais se repetiram nos estudos, dois não apresentam essa versão. O coeficiente Kappa mostrou concordância entre moderada e boa $(p<0,001)$.
Este estudo apresentou um panorama da ligação de conteúdo de instrumentos da prática clínica à CIF identificando quais têm versão em língua portuguesa, o que contribuirá para o potencial fortalecimento da abordagem biopsicossocial dos profissionais de saúde.

Descritores | Classificação Internacional de Funcionalidade, Incapacidade e Saúde; CIF; Questionários; Revisão.

\begin{abstract}
I Clinical practice has been subsidized by instruments that record and allow access to information on functionality and health of individuals. One way to approach the content of the International Classification of Functioning, Disability and Health (ICF) is to use it as a reference to aid the professional in choosing the most appropriate instrument to access biopsychosocial information. We intend to identify health assessment instruments that had their content linked to the contents of the ICF and the presence of the Portuguese version of them. A systematic review was conducted of the SciELO Brazil, Lilacs and PubMed databases with the descriptors "ICF", "questionnaire" and "linking rules" in Portuguese and English. Three independent researchers performed the papers' selection, and the level of agreement was obtained by the kappa coefficient. The eligibility criteria were primary studies for questionnaires, scales, indices and checklists content linking to ICF published after 2001 in
\end{abstract}

Este estudo foi desenvolvido no Laboratório de Informática em Saúde do PPGTS da PUCPR na cidade de Curitiba (PR), Brasil. IPontifícia Universidade Católica do Paraná (PUCPR) - Curitiba (PR), Brasil. E-mail: karoleen.s@hotmail.com. Orcid: 0000-0002-4420-3171 ${ }^{2}$ Pontifícia Universidade Católica do Paraná (PUCPR) - Curitiba (PR), Brasil. E-mail: rafaellasb@yahoo.com.br. Orcid: 0000-0002-4613-0834 ${ }_{3}^{3}$ Pontifícia Universidade Católica do Paraná (PUCPR) - Curitiba (PR), Brasil. E-mail: katren.correa@gmail.com. Orcid: 0000-0002-0175-2728 ${ }_{4}^{4}$ Pontifícia Universidade Católica do Paraná (PUCPR) - Curitiba (PR), Brasil. E-mail: auristela.lima@gmail.com. Orcid: 0000-0001-5086-0701 
Portuguese or English. A total of 61 articles were included, of which 19 were of Brazilian origin. Given the totality of 250 instruments with ICF-related content, 158 (63.2\%) presented a Portuguese-language version; just two of the 37 most cited works presented a Portuguese version. The Kappa coefficient showed significative agreement between moderate and good. This study provided an overview of the content connection between clinical practice instruments to ICF, identifying which instruments have Portuguese translation and its potential for strengthening the biopsychosocial approach. Keywords | International Classification of Functioning, Disability and Health; ICF; Questionnaires; Review.

RESUMEN | La práctica clínica ha sido subsidiada por instrumentos que permiten acceder y registrar informaciones de funcionalidad y salud de los individuos. Una forma de conocer qué contenidos de la Clasificación Internacional de Funcionalidad, Incapacidad y Salud (CIF) los instrumentos contemplan es usarla como referencia para auxiliar al profesional en la elección del más adecuado para acceder a informaciones biopsicosociales. Lo objetivo fue identificar los instrumentos de la práctica clínica en la salud que tuviesen su contenido vinculado con el CIF y la disponibilidad de la versión en el idioma portugués. Para ello se realizó una revisión sistemática en las bases de datos SciELO Brasil, Lilacs y PubMed con las palabras clave "CIF", "cuestionario" y "reglas de vinculación "en portugués e inglés. Tres investigadores independientes realizaron la selección, y el nivel de concordancia fue obtenido por el coeficiente Kappa. Los criterios de elegibilidad fueron: estudios primarios de vinculación del contenido de cuestionarios, escalas, índices y listas de control con CIF publicados después de 2001 en el idioma portugués o Ingles. Fueron incluidos 61 artículos, siendo 19 de origen brasilero. De los 250 instrumentos con contenido relacionado a CIF, 158 (63,2\%) presentaron versión en portugués, siendo que de los 37 que más se repitieron en los estudios dos no presentaron esta versión. El coeficiente Kappa mostró concordancia entre moderada y buena $(p<0,001)$. Se ha delineado un panorama de la vinculación del contenido de instrumentos de la práctica clínica a la CIF identificando cuáles tienen versión en la lengua portuguesa y su potencial fortalecimiento del enfoque biopsicosocial. Palabras clave | Clasificación Internacional del Funcionamiento, de la Discapacidad y de la Salud; CIF; Cuestionarios; Revisión.

\section{INTRODUÇÃO}

O aumento da prevalência de condições crônicas em saúde e do envelhecimento populacional tem impactado na morbidade, funcionalidade e mortalidade da população mundial no século XXI. A manutenção da saúde, portanto, tem sido um grande objetivo para a sociedade moderna ${ }^{1-3}$.

O termo funcionalidade sintetiza a operacionalização da saúde biológica e da saúde vivida e foi reconhecido pela Organização Mundial da Saúde (OMS) como o terceiro indicador da saúde, juntamente com a mortalidade e a morbidade ${ }^{3}$.

Os registros de informações em saúde por meio de instrumentos da prática clínica como questionários, escalas, índices, checklists e outros têm sido utilizados para subsidiar o acompanhamento das mudanças no perfil de saúde da população. Tais registros subsidiam a investigação do estado de saúde, avaliam o impacto das condições de saúde na vida do indivíduo e auxiliam no delineamento dos planos de tratamento e demais intervenções, acompanhando os desfechos ${ }^{4}$.

Outra forma de registrar as informações é por meio de classificações, que utilizam uma estrutura mundialmente aceita e contemplam diferentes domínios em saúde adotando como referência para uma linguagem unificada e consistente para profissionais de diferentes áreas ${ }^{5}$. $\mathrm{O}$ registro de informações sobre funcionalidade, tem sido recomendado com o uso da Classificação Internacional de funcionalidade, Incapacidade e Saúde (CIF), publicada em 2001 pela Organização Mundial da Saúde; e a morbidade e mortalidade, pela Classificação Estatística Internacional de Doenças e Problemas Relacionados à Saúde ${ }^{1,3,6-8}$.

O uso conjunto dessas duas formas de coleta de dados permite a comparação dos registros em saúde, o que pode garantir que uma ampla gama de informações esteja disponível de uma maneira consistente para profissionais de saúde e gestores nos diferentes níveis de atenção à saúde ${ }^{4}$. Assim, disponibilizar o conteúdo dos instrumentos poderá contribuir para a escolha daquele que melhor contemple os conteúdos demandados pelo contexto, pelo indivíduo que está sendo atendido e pelo próprio profissional. A CIF provê uma linguagem comum para a prática clínica e tornou-se uma referência para a comparação de conteúdo de instrumentos já existentes, bem como para aqueles em desenvolvimento por conter um conjunto exaustivo de categorias que se relacionam à funcionalidade humana nos domínios Funções do Corpo, Estruturas do Corpo, Atividades e Participação do indivíduo, bem como a dos Fatores Ambientais ${ }^{4,9,10}$. 
Cieza et al. ${ }^{4}$ desenvolveram uma abordagem sistemática e padronizada para ligar o conteúdo de instrumentos da prática clínica com a CIF. Nesse estudo, os autores desenvolveram regras de ligação de conteúdo e testaram sua confiabilidade para estes instrumentos da prática clínica. Posteriormente publicaram sua atualização em $2005^{10}$, estendendo a aplicação das regras para outras formas de coleta de informações em saúde como testes laboratoriais, de imagem e outros exames clínicos, e as refinaram em $2016^{9}$ para fortalecer a transparência do processo de ligação.

Tal ligação usando a CIF como referência permite a seleção de categorias representativas para dado contexto ou situação ${ }^{9,11}$, auxilia o profissional na escolha do instrumento mais adequado para acessar as informações sobre a capacidade funcional dos indivíduos ${ }^{9}$, pois os instrumentos são fontes de informação para operacionalizar o modelo proposto pela CIF e ao mesmo tempo são subsídios para o conhecimento da cobertura deles sobre o modelo biopsicossocial.

$\mathrm{Na}$ literatura brasileira, relata-se crescente produção científica com a CIF ${ }^{12-15}$, entretanto, ainda há carência em se conhecer os instrumentos já ligados à CIF para que se possam operacionalizar tais conteúdos contemplados no modelo biopsicossocial proposto pela OMS.

Diante desse cenário, a presente revisão objetivou identificar instrumentos da prática clínica em saúde que tiveram seu conteúdo ligado com a CIF e a presença de sua versão na língua portuguesa.

\section{METODOLOGIA}

Trata-se de uma revisão sistemática realizada no período de Fevereiro a Março de 2017 e atualizada em março de 2019 que seguiu as recomendações do Preferred Reporting Items for Systematic Reviews and Meta-Analysis ${ }^{16} \mathrm{em}$ ambos os momentos de busca quando pertinente.

A busca dos estudos foi realizada nas línguas portuguesa e inglesa. A decisão pela busca na língua inglesa justifica-se pelo fato da CIF ser uma linguagem universal, e as categorias ligadas para um instrumento em outra lingua poderão ser consideradas para a respectiva versão na língua portuguesa, podendo ser customizadas de acordo com o contexto, a perspectiva e a necessidade de cada profissional.

As bases de dados acessadas foram Scientific Eletronic Library Online (SciELO Brasil), Latin American and Caribbean Health Science (Lilacs) e Medical Literature Analysis and Retrieval System Online/U.S. National
Library of Medicine (Medline/PubMed) e os descritores estão listados no Quadro 1.

Quadro 1. Descritores usados para busca dos artigos nas bases eletrônicas

\begin{tabular}{|c|c|}
\hline Bases & Descritores \\
\hline Lilacs & $\begin{array}{l}\text { 10 estratégia: questionnaires or questionnaire or survey } \\
\text { or surveys [words] and International Classification of } \\
\text { Functioning, Disability and Health or ICF [words] } \\
2^{2} \text { estratégia: International Classification of Functioning, } \\
\text { Disability and Health or ICF [words] and Linking rules or } \\
\text { linking or linking and rule [words] }\end{array}$ \\
\hline $\begin{array}{l}\text { Medline/ } \\
\text { PubMed }\end{array}$ & $\begin{array}{l}\text { ((ICF[All Fields] OR ("international classification of } \\
\text { functioning, disability and health"[MeSH Terms] OR } \\
\text { ("international”[All Fields] AND "classification"[All } \\
\text { Fields] AND "functioning"[All Fields] AND } \\
\text { "disability"[All Fields] AND "health"[All Fields]) OR } \\
\text { "disability and health international classification of } \\
\text { functioning"[All Fields] OR ("international”"[All Fields] } \\
\text { AND "classification"[All Fields] AND "functioning"[All } \\
\text { Fields] AND "disability"[All Fields] AND "health"[All } \\
\text { Fields]) OR "international classification of functioning, } \\
\text { disability and health"[All Fields])) AND ("surveys } \\
\text { and questionnaires"[MeSH Terms] OR ("surveys"[All } \\
\text { Fields] AND "questionnaires"[All Fields]) OR "surveys } \\
\text { and questionnaires"[All Fields] OR "questionnaire"[All } \\
\text { Fields])) AND (linking[All Fields] AND rules[All Fields]) }\end{array}$ \\
\hline SciELO & $\begin{array}{l}\text { ((questionnaires) OR (questionnaire) OR (survey) OR } \\
\text { (surveys) OR (surveys AND questionnaires)) AND } \\
\text { (((international classification of functioning, disability } \\
\text { AND health) OR (icf))) OR (((international classification } \\
\text { of functioning, disability AND health) OR (icf))) AND } \\
\text { (((linking) AND (rules)) OR (linking) OR (linking rules))) }\end{array}$ \\
\hline
\end{tabular}

A busca dos estudos nas bases SciELO Brasil e Lilacs foram realizada a partir da associação dos descritores "CIF/ICF", "questionário/questionnaire" e "regra de ligação/linking rules”, e, em seguida, utilizando os descritores separadamente. Para complementar a busca foi acessada a base Medline/PubMed com a associação dos três descritores acima citados.

A seleção dos estudos seguiu os critérios: língua portuguesa, inglesa ou espanhola; estudo primário de ligação de conteúdo de questionários, escalas, índices e checklists utilizados na prática clínica em saúde com o conteúdo da CIF; publicado após o estabelecimento da primeira versão da CIF em 2001 ${ }^{17}$; com instrumentos para avaliação de pessoas com idade maior a 18 anos.

Não se estabeleceu critério de inclusão para a área de estudo nem para o método usado na ligação dos instrumentos com a CIF, para levantar formas utilizadas para a realização da ligação. Foram excluídos artigos duplicados, artigos teóricos de desenvolvimento das regras de ligação, artigos que não explicitaram quais instrumentos foram ligados (nome do instrumento), artigos exclusivamente de desenvolvimento de instrumentos e artigos que ligassem exclusivamente inquéritos e 
inventários de saúde, testes clínicos, exames laboratoriais e de imagem, prontuários eletrônicos, classificações e/ou scores preditores de mortalidade.

Foram incluídos estudos que também ligaram à CIF inquéritos e inventários, testes, exames, prontuários, classificações e/ou scores preditores de mortalidade, porém, de tais estudos só foram apresentados os resultados de ligação da CIF com os instrumentos de interesse.

A seleção dos artigos foi feita de forma independente e cega por três pesquisadores. Foram lidos os títulos e subsequentemente os resumos para a eleição dos a serem lidos na íntegra.

O nível de concordância entre os pesquisadores para a elegibilidade dos estudos foi verificado pelo coeficiente Kappa estatístico, sendo que os valores do Kappa variam de 0 a 1, com valores abaixo de 0,20 indicando concordância pobre, entre 0,21 e 0,40 fraca, entre 0,41 e 0,61 indicando concordância moderada, entre 0,61 e 0,80 concordância boa e acima de 0,81 muito boa ${ }^{18}$.

Houve busca manual de estudos que tratassem de ligação de instrumentos com a CIF nas referências de revisões sistemáticas e em estudos que ligaram alguns instrumentos e utilizaram o resultado de instrumentos já ligados por outros pesquisadores.

Foram extraídos os seguintes dados dos estudos: base indexadora, autores, ano de publicação, instrumentos da prática clínica em saúde que foram ligados à CIF, procedimentos metodológicos de ligação adotados, componentes da CIF contemplados nos instrumentos e contextos nos quais os estudos foram realizados e se o estudo realizou a classificação das categorias da CIF selecionadas a partir da ligação de conteúdo do instrumento. Quando houve dúvida nos registros um consenso entre os pesquisadores foi estabelecido.

Para os instrumentos advindos da língua inglesa foi realizada uma busca manual na literatura para identificar quais apresentavam versão na língua portuguesa. Também se verificou manualmente a compatibilidade direta de opção de resposta dos instrumentos com a opção de resposta da CIF, ou seja, se todos os itens dos instrumentos apresentavam cinco opções de respostas graduadas ordinalmente. As demais opções de resposta identificadas foram consideradas como não diretas.

\section{RESULTADOS}

Sessenta e um estudos primários de ligação de conteúdo dos instrumentos da prática clínica em saúde com a CIF foram incluídos nesta revisão (Figura 1), sendo que 50 originaram-se de busca eletrônica. Os 11 demais advieram de busca manual, em que 6 foram de artigos de revisão ${ }^{5,13-15,19}$, dois deles ${ }^{20,21}$ pertencentes à revisão de Faria et al. ${ }^{19}$ e quatro ${ }^{22-25}$ à revisão de Fayed et al. ${ }^{5}$. As outras 3 revisões ${ }^{13-15}$, também tiveram suas referências verificadas, porém, os estudos primários de ligação incluídos nelas já haviam sido capturados na busca eletrônica. Os cinco demais estudos incluídos a partir da busca manual advieram de citações em estudos de ligação primária na busca eletrônica ${ }^{26-30}$.

O coeficiente Kappa mostrou nível de concordância de moderado a bom ( $\mathrm{p}<0,001)$ entre os três pesquisadores, expressado por duplas de pesquisadores os valores mínimo e máximo encontrados de 0,488 (85,2\%) e 0,651 (90,6\%).

Foram identificados 250 diferentes instrumentos, dos quais $158(63,2 \%)$ apresentam versão na língua portuguesa. A quantidade de instrumentos ligados por cada um dos estudos diferiu, sendo que 37 (60,6\%) estudos ligaram conteúdo de 2 a 6 instrumentos à CIF, $16(26,2 \%)$ ligaram um, $8(13,1 \%)$ ligaram de 10 a 20 e um único estudo ligou 59.

Trinta e sete instrumentos foram ligados por mais de um estudo. O SF-36 foi ligado por 11 dos 61 estudos; FIM por 8; DASH por 7; BI e NHP por 6 estudos cada; EQ-5D, MMSE e SSQol por 4; DRS, FAI, GOS, HADS, SIP e WHOQOL-BREAF por 3 e ABC, BBS, BDI, CIQ, COPM, EORTCQLQ-C30, FAM, FSS, HRSD, MAS, MI, mRS, NEADL, NIHSS, QuickDASH, RMA, RMI, RS, SA-SIP30, SIS, SPADI, SSQ, SSS, WHOdas 2.0 e o WAIS-R por 2 estudos cada um. Desses, o NEADL e o SIP não apresentaram versão em português. Também não apresentou versão em português o CDIP-58.

Quanto a metodologia de ligação, 1 estudo utilizou apenas o refinamento das regras de ligação ${ }^{9}, 2$ estudos utilizaram as regras propostas por Cieza et al. ${ }^{4}$ e Cieza et al. ${ }^{10}$ e seu refinamento 9 , 78,7\% $(n=48)$ dos estudos utilizaram as regras propostas por Cieza et al. ${ }^{4}$ e Cieza et al. ${ }^{10} \mathrm{e}$ os demais utilizaram consenso ou outras metodologias. Além disso, a maioria dos estudos estavam inseridos no contexto de Neurologia e Ortopedia. Do total dos estudos, 19 foram publicações brasileiras.

O componente Atividades e Participação (d) foi o mais contemplado $(93,4 \%, n=57)$ nos estudos, seguido por Funções do Corpo $(81,9 \%, n=50)$, Fatores Ambientais $(55,7 \% \mathrm{n}=34)$ e, Estruturas do Corpo $(16,3 \%, n=10)$. Seis estudos contemplaram todos os componentes da CIF. 


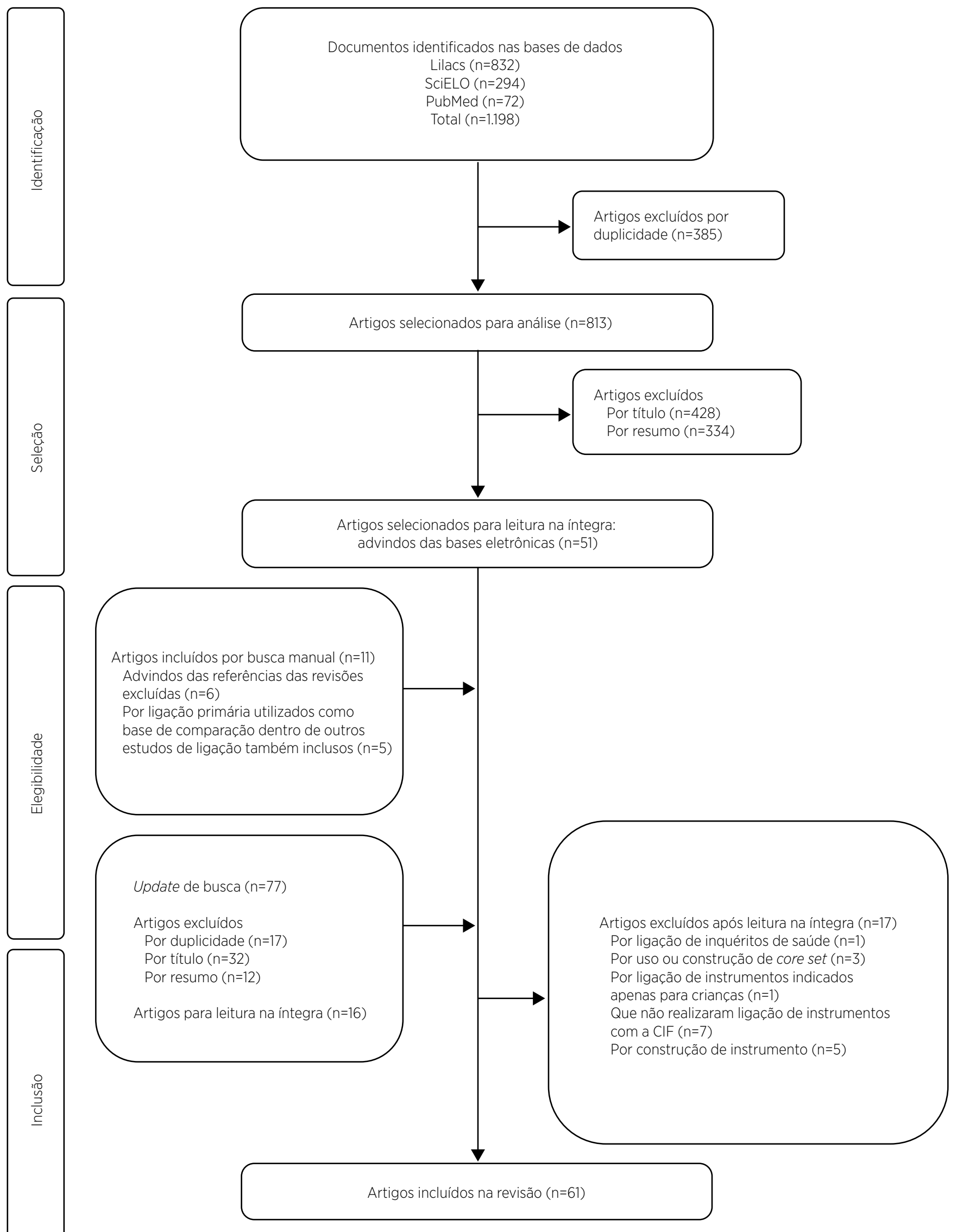

Figura 1. Fluxograma de busca, seleção e inclusão dos artigos 
Quanto à forma de expressar os resultados, houve estudos que demonstraram a ligação de cada item dos instrumentos à cada categoria CIF selecionada ${ }^{31-55}$. Philbois et al. ${ }^{56}$ e Gomes et al. ${ }^{57}$ ligaram os itens dos instrumentos aos componentes da CIF. Campos et al..$^{58}$ também demonstraram a ligação de cada item dos instrumentos às categorias $\mathrm{CIF}$, porém, tais categorias advieram do core set para AVE e não da CIF completa.

Outros estudos apresentaram as categorias da CIF que foram ligadas a cada instrumento, sem especificar a qual item a categoria se referia ${ }^{10,20,21,25,27,28,30,59-71}$. Carvalho et al. ${ }^{62}$ também demonstraram as categorias, porém, tais categorias advieram do core set para câncer de mama.

Ainda, estudos que realizaram a ligação de mais de um instrumento expressaram como resultado a ligação de cada item a cada categoria para um dos instrumentos ligados ${ }^{72-75}$.

Alguns estudos deram enfoque para apenas um componente da CIF. Alvarelhão et al. ${ }^{76}$ e Guscia et al. ${ }^{23}$ demonstraram o número de categorias de Fatores Ambientais ligadas a cada instrumento.Já Dixon et al. ${ }^{26}$, Moura et al. ${ }^{54} \mathrm{e}$ Prodinger et al. ${ }^{69}$ demonstraram o número de categorias de Atividades e Participação. van der Mei et al. ${ }^{77}$ apresentaram, além das categorias de Atividades e Participação, o porcentual de categorias identificadas nos demais componentes. Roe et al. ${ }^{29}$ apresentaram os capítulos de cada componente contidos nos instrumentos.

Fréz et al. ${ }^{78}$ ligaram as categorias da CIF aos oito domínios do questionário SF-36. Geyh et al. ${ }^{22}$ e Scheuringer et al. ${ }^{24}$ apresentaram as categorias e suas frequências de aparecimento para o total de instrumentos ligados. De Pauw et al. ${ }^{79}$ apresentaram de forma similar, porém apresentaram a frequência dos capítulos de Função do Corpo e Atividade e Participação.

Dos 250 instrumentos identificados 17,2\% ( $\mathrm{n}=43)$ apresentou compatibilidade direta da opção de resposta de todos os itens do instrumento com a CIF, e a maior parte $(82,8 \% n=207)$ compatibilidade não direta.

Quanto à classificação das categorias da CIF apenas 6 estudos, todos brasileiros, utilizaram os qualificadores40,49,53,54,61,78.

Os dados aqui apresentados estão resumidos no Quadro 2.

Quadro 2. Artigos incluídos na busca, seu país de origem e contextos, bem como os instrumentos que tiveram conteúdos ligados à CIF, procedimento metodológico utilizado para tal e componentes da CIF contemplados pela ligação em cada um dos artigos

\begin{tabular}{|c|c|c|c|c|}
\hline Autor (país) & Contexto & Instrumentos que tiveram o conteúdo ligado à CIF & Procedimento de ligação & Componentes CIF \\
\hline $\begin{array}{l}\text { Dahlgren et al. }{ }^{36} \\
\text { (Suécia) }\end{array}$ & \multirow{6}{*}{$\begin{array}{l}\frac{0}{7} \\
\frac{0}{0} \\
\frac{0}{3} \\
\frac{0}{2}\end{array}$} & KB (Klein-Bell Activities of Daily Living Scale) & Cieza et al., 2005 & $b, d, e$ \\
\hline $\begin{array}{l}\text { Fréz et al. }{ }^{39} \\
\text { (Brasil) }\end{array}$ & & FIM (Functional Independence Measure)* & Cieza et al., 2005 & $b, d, e$ \\
\hline $\begin{array}{l}\text { Silva et al. }{ }^{4 \#} \\
\text { (Brasil) }\end{array}$ & & $\begin{array}{l}\text { NHP (Nottingham Health Profile)* } \\
\text { SSQoL (Stroke Specific Quality of Life Scale)*+ }\end{array}$ & Cieza et al., 2005 & $d$ \\
\hline $\begin{array}{l}\text { Koopman et al. }{ }^{41} \\
\text { (Holanda) }\end{array}$ & & $\begin{array}{l}\text { FSS (Fatigue Severity Scale)* } \\
\text { CIS20-F (Checklist Individual Strength- sub-scale } \\
\text { subjective experience of fatigue)* }\end{array}$ & Cieza et al., 2005 & $b, d$ \\
\hline $\begin{array}{l}\text { Berzina et al. }{ }^{32} \\
\text { (Suíça) }\end{array}$ & & mRS (The Modified Rankin Scale)* & Cieza et al., 2005 & $b, d, e$ \\
\hline $\begin{array}{l}\text { Prodinger et al. }{ }^{69} \\
\text { (Alemanha) }\end{array}$ & & $\begin{array}{l}\text { SF-36 (Short Form Health Survey)* } \\
\text { WHODAS } 2.0 \text { (WHO Disability Schedule 2.0)* } \\
\text { SIS } 3.0 \text { (Stroke Impact Scale 3.0)*+ }\end{array}$ & $\begin{array}{l}\text { Cieza et al., 2002; } \\
\text { Cieza et al., 2005; } \\
\text { Cieza et al., } 2016\end{array}$ & $d$ \\
\hline $\begin{array}{l}\text { Sigl et al. }{ }^{67} \\
\text { (Alemanha) }\end{array}$ & \multirow{5}{*}{ 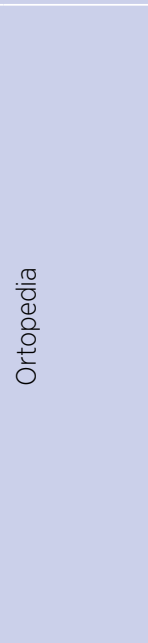 } & $\begin{array}{l}\text { BASFI (Bath Ankylosing Spondylitis Functional Index)* } \\
\text { DFI (Dougados Functional Index)* } \\
\text { HAQ-S (Health Assessment Questionnaire modified for the } \\
\text { spondyloarthropathies)* } \\
\text { RLDQ (Revised Leeds Disability Questionnaire)* }\end{array}$ & Cieza et al., 2002 & $b, d, e$ \\
\hline $\begin{array}{l}\text { Sigl et al. }{ }^{73} \\
\text { (Alemanha) }\end{array}$ & & $\begin{array}{l}\text { NASS (North American Spine Society Lumbar Spine Outcome } \\
\text { Assessment Instrument) } \\
\text { ODI (Oswestry Low Back Disability Questionnaire)*+ } \\
\text { RMDQ (Roland Morris Disability Questionnaire)* }\end{array}$ & Cieza et al., 2002 & $b, d, e$ \\
\hline $\begin{array}{l}\text { Drummond et al. }{ }^{38} \\
\text { (Brasil) }\end{array}$ & & DASH (Disabilities of the Arm, Shoulder and Hand)* ${ }^{+}$ & Cieza et al., 2005 & $b, d$ \\
\hline $\begin{array}{l}\text { Dixon et al. }{ }^{26} \\
\text { (Escócia) }\end{array}$ & & DASH (Disabilities of the Arm, Shoulder and Hand) ${ }^{*+}$ & $\begin{array}{l}\text { Nível de concordância } \\
\text { entre avaliadores }\end{array}$ & $d$ \\
\hline $\begin{array}{l}\text { Alviar et al. }{ }^{60} \\
\text { (Austrália) }\end{array}$ & & $\begin{array}{l}\text { OHS (Oxford Hip Score) }{ }^{\dagger} \\
\text { OKS (Oxford Knee Score) }{ }^{*} \\
\text { HOOS (Hip Dysfunction and Osteoarthritis Outcome Score) }{ }^{+} \\
\text {KOOS (Knee Injury and Osteoarthritis Outcome Score) } \\
\text { AIMS (Arthritis Impact Measurement Scales) }{ }^{*}+\end{array}$ & Cieza et al., 2005 & $b, s, d, e$ \\
\hline
\end{tabular}


Quadro 2. Continuação

\begin{tabular}{|c|c|c|c|c|}
\hline Autor (país) & Contexto & Instrumentos que tiveram o conteúdo ligado à CIF & Procedimento de ligação & Componentes CIF \\
\hline $\begin{array}{l}\text { Arumugam et al. }{ }^{31} \\
\text { (Canadá) }\end{array}$ & & WLQ-26 (26-Item Work Limitations Questionnaire)* † & Cieza et al., 2005 & $b, d, e$ \\
\hline $\begin{array}{l}\text { Roe et al. }{ }^{29} \\
\text { (Noruega) }\end{array}$ & $\begin{array}{l}\frac{0}{0} \\
\frac{0}{0} \\
\frac{0}{0} \\
\frac{1}{0}\end{array}$ & $\begin{array}{l}\text { Constant (Constant Murley shoulder Score) } \\
\text { ASES (American Shoulder and Elbow Surgeons Standardized } \\
\text { form for Assessment of the Shoulder)* } \\
\text { UCLA (University of California at } \\
\text { LOS Angeles shoulder rating Scale)* } \\
\text { DASH (Disability of the Arm, Shoulder and Hand)* † } \\
\text { SST (Simple Shoulder Test)* } \\
\text { SPADI (Shoulder Pain and Disability Index)* } \\
\text { WORC (Western Ontario Rotator Cuff Index)* } \\
\text { SRQ (Shoulder Rating Questionnaire)* } \\
\text { SDQ (Shoulder Disability Questionnaire) } \\
\text { OSS (Oxford Shoulder Score)* } \\
\text { WOSI (Western Ontario Shoulder Instability Index)* } \\
\text { QuickDASH (Shortened version of Disabilities of the Arm, } \\
\text { Shoulder and Hand)* } \\
\text { Penn (Penn shoulder score)* } \\
\text { SF-36 (36-item Short-Form Health Survey)* } \\
\text { SF-12 (12-Item Short-Form Health Survey)* } \\
\text { JCQ (Job Content Questionnaire)* } \\
\text { Nordic (Standardized Nordic questionnaires for the analysis of } \\
\text { musculoskeletal symptoms)* } \\
\text { EQ-5D (European Quality of Life Instrument-5D)* } \\
\text { FABQ (Fear-Avoidance Beliefs Questionnaire)* } \\
4 \text { DSQ (Four-Dimensional Symptom Questionnaire) }\end{array}$ & Cieza et al., 2005 & $b, s, d, e$ \\
\hline $\begin{array}{l}\text { Fréz et al. }{ }^{78 \#} \\
\text { (Brasil) }\end{array}$ & & SF-36 (36-item Short-Form Health Survey)* & Consenso & $b, s, d$ \\
\hline $\begin{array}{l}\text { Forget e Higgins }{ }^{64} \\
\text { (Canadá) }\end{array}$ & & $\begin{array}{l}\text { DASH (Disabilities of the Arm, Shoulder and Hand)* } \\
\text { QuickDASH (shortened version of Disabilities of the Arm, } \\
\text { Shoulder and Hand)* } \\
\text { PRWE (Patient rated wrist evaluation) } \\
\text { PRWHE (Patient rated wrist/hand evaluation) } \\
\text { PEM (Patient Evaluation Measure) } \\
\text { MHQ (MHQ Michigan Hand Questionnaire) }{ }^{\dagger} \\
\text { UEFI (Upper extremity functional index)* }\end{array}$ & Cieza et al., 2005 & $b, d$ \\
\hline $\begin{array}{l}\text { Vincent et al. } .^{51} \\
\text { (Canadá) }\end{array}$ & & $\begin{array}{l}\text { PREE (Patient Rated Elbow Evaluation)* } \\
\text { pASES-e (American Shoulder and } \\
\text { Elbow Surgeons Society - Elbow Form)* }\end{array}$ & Cieza et al., 2005 & $b, d$ \\
\hline $\begin{array}{l}\text { Nicol et al. }{ }^{45} \\
\text { (Suíça) }\end{array}$ & & $\mathrm{BQ}$ (Bournemouth Questionnaire)* & Cieza et al., 2005 & $b, d$ \\
\hline $\begin{array}{l}\text { Philbois et al. }{ }^{56} \\
\text { (Brasil) }\end{array}$ & $\begin{array}{l}\frac{-0}{\overline{0}} \\
\frac{0}{0} \\
\frac{0}{0}\end{array}$ & $\begin{array}{l}\text { SPADI (Shoulder Pain and Disability Index)* } \\
\text { DASH (Disabilities of the Arm, Shoulder and Hand)*+ }\end{array}$ & $\begin{array}{l}\text { Nivel de concordância } \\
\text { entre avaliadores }\end{array}$ & $b, s, d$ \\
\hline $\begin{array}{l}\text { Fréz et al. }{ }^{65} \\
\text { (Brasil) }\end{array}$ & ఫ & $\begin{array}{l}\text { AMP (Amputee Mobility Predictor)* } \\
\text { PEQ (Prosthesis Evaluation Questionnaire)* } \\
\text { HAI (Hill Assessment Index) } \\
\text { SAI (Stair Assessment Index) }\end{array}$ & Cieza et al., 2005 & e \\
\hline $\begin{array}{l}\text { De Pauw et al. }{ }^{99} \\
\text { (Bélgica) }\end{array}$ & & $\begin{array}{l}\text { TWSTRS (Toronto Western Spasmodic Torticollis Rating Scale) }{ }^{*} \\
\text { CDIP-58 (Cervical Dystonia Impact Profile) }{ }^{\dagger}\end{array}$ & $\begin{array}{l}\text { Cieza et al., } 2002 \\
\text { Cieza et al., } 2005\end{array}$ & $b, d$ \\
\hline $\begin{array}{l}\text { Weigl et al. }{ }^{52} \\
\text { (Alemanha) }\end{array}$ & & $\begin{array}{l}\text { WOMAC (Western Ontario and McMaster Universities)* } \\
\text { LAI (Lequesne Algofunctional Index)* }\end{array}$ & Cieza et al., 2005 & $b, d, e$ \\
\hline $\begin{array}{l}\text { Stamm et al. }{ }^{75} \\
\text { (Áustria) }\end{array}$ & & $\begin{array}{l}\text { COPM (Canadian Occupational Performance Measure)* } \\
\text { AMPS (Assessment of Motor and Process Skills) } \\
\text { SODA (Sequential Occupational Dexterity Assessment) }\end{array}$ & Cieza et al., 2002 & $b, d$ \\
\hline $\begin{array}{l}\text { Stucki e Cieza } \\
\text { (Alemanha) }\end{array}$ & $\begin{array}{l}\frac{0}{\pi} \\
\frac{0}{0} \\
\frac{0}{0} \\
\frac{0}{0}\end{array}$ & $\begin{array}{l}\text { HAQ-DI (Health Assessment Questionnaire Disability Index)* } \\
\text { AIMS2 (Arthritis Impact Measurement Scales 2)*+ } \\
\text { SF-36 (36-item Short-Form Health Survey)* }\end{array}$ & Cieza et al., 2002 & $b, d$ \\
\hline $\begin{array}{l}\text { Stamm et al. }{ }^{74} \\
\text { (Áustria) }\end{array}$ & 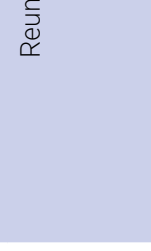 & $\begin{array}{l}\text { HAQ (Health Assessment Questionnaire)* } \\
\text { AUSCAN Index* } \\
\text { Cochin Scale (Cochin Rheumatoid Hand Disability Scale)* } \\
\text { FIHOA (Functional Index of Hand Osteoarthritis)* } \\
\text { SACRAH (Score for Assessment and Qualification of Chronic } \\
\text { Rheumatoid Affections of the Hands Questionnaire) } \\
\text { AIMS2-SF (Arthritis Impact Measurement } 2 \text { Scales short form)*+ }\end{array}$ & Cieza et al., 2005 & $b, d, e$ \\
\hline
\end{tabular}


Quadro 2. Continuação

\begin{tabular}{|c|c|c|c|c|}
\hline Autor (país) & Contexto & Instrumentos que tiveram o conteúdo ligado à CIF & Procedimento de ligação & Componentes CIF \\
\hline $\begin{array}{l}\text { Prodinger et al. } .^{72} \\
\text { (Áustria) }\end{array}$ & 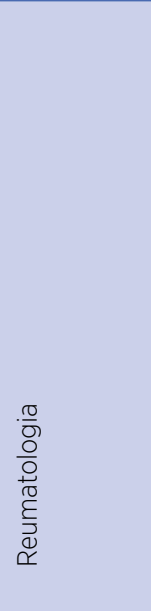 & $\begin{array}{l}\text { FIQ (Fibromyalgia Impact Questionnaire)* } \\
\text { MPQ (McGill Pain Questionnaire)* } \\
\text { SF-MPQ (Short Form McGill Pain Questionnaire)* } \\
\text { BPI (Brief Pain Inventory)* } \\
\text { LANSS (Leeds Assessment of Neuropathic Symptoms and } \\
\text { Signs Pain Scale)* } \\
\text { FACIT-FS (Functional Assessment of Chronic Illness Therapy)*+ } \\
\text { FACIT-F (Functional Assessment of Chronic Illness Therapy- } \\
\text { Fatigue)*t } \\
\text { FSS (Fatigue Severity Scale)* } \\
\text { MFI-20 (Multidimensional Fatigue Inventory)* } \\
\text { MAF (Multidimensional Assessment of Fatigue) } \\
\text { MOS (Sleep scale from Medical Outcomes Study) } \\
\text { SF-36 (36-item Short-Form Health Survey)* } \\
\text { ASEX (Arizona Sexual Experiences Scale)* } \\
\text { BDI (Beck Depression Inventory)* } \\
\text { HADS (Hospital Anxiety and Depression Scale)* } \\
\text { HRSD (Hamilton Rating Scale for Depression)* }\end{array}$ & Cieza et al., 2005 & $b, s, d, e$ \\
\hline $\begin{array}{l}\text { Rat et al. }{ }^{47} \\
\text { (França) }\end{array}$ & & OAKHQOL (Osteoarthritis Knee and Hip Quality of Life) & Cieza et al., 2005 & $b, d, e$ \\
\hline $\begin{array}{l}\text { Milman et al. }{ }^{66} \\
\text { (Canadá) }\end{array}$ & & $\begin{array}{l}\text { BVAS (Birmingham Vasculitis Activity Score)* } \\
\text { BVASv3 (Birmingham Vasculitis Activity Score version 3)* } \\
\text { BVAS/WG (Birmingham Vasculitis Activity Score for } \\
\text { granulomatosis with polyangiitis)* } \\
\text { VDI (Vasculitis Damege Index) } \\
\text { SF-36 (36-item Short-Form Health Survey)* }\end{array}$ & Cieza et al., 2005 & $b, s, d$ \\
\hline $\begin{array}{l}\text { Moura et al. }{ }^{54 \#} \\
\text { (Brasil) }\end{array}$ & & SALSA (Screening of Activity Limitation \& Safety Awareness)* & Não explicitado & $d$ \\
\hline $\begin{array}{l}\text { Bladh et al. }{ }^{33} \\
\text { (Suécia) }\end{array}$ & $\begin{array}{l}\frac{0}{\circ} \\
\frac{0}{0} \\
\frac{0}{0} \\
\frac{0}{0} \\
\frac{1}{2}\end{array}$ & $\begin{array}{l}\text { FES-I (Falls Efficacy Scale-International) }{ }^{\dagger} \\
\text { FES(S) (Swedish version of the Falls Efficacy Scale) }{ }^{+} \\
\text {ABC (Activities-specific Balance Confidence Scale)* } \\
\text { SAFFE (modified Survey of Activities and Fear of Falling in the } \\
\text { Elderly) }{ }^{\dagger}\end{array}$ & Cieza et al., 2005 & $b, d$ \\
\hline $\begin{array}{l}\text { Marques et al. }{ }^{43} \\
\text { (Portugal) }\end{array}$ & $\circlearrowleft$ & EASY-Care Standard* & Cieza et al., 2005 & $b, s, d, e$ \\
\hline $\begin{array}{l}\text { Dahlgren et al. }{ }^{36} \\
\text { (Suécia) }\end{array}$ & & KB (Klein-Bell Activities of Daily Living Scale) & Cieza et al., 2005 & $b, d, e$ \\
\hline $\begin{array}{l}\text { Fréz et al. }{ }^{39} \\
\text { (Brasil) }\end{array}$ & & FIM (Functional Independence Measure)* & Cieza et al., 2005 & $b, d, e$ \\
\hline $\begin{array}{l}\text { Silva et al. }{ }^{49 \#} \\
\text { (Brasil) }\end{array}$ & $\frac{\pi}{\pi}$ & $\begin{array}{l}\text { NHP (Nottingham Health Profile)* } \\
\text { SSQoL (Stroke Specific Quality of Life Scale)*+ }\end{array}$ & Cieza et al., 2005 & $d$ \\
\hline $\begin{array}{l}\text { Koopman et al. }{ }^{41} \\
\text { (Holanda) }\end{array}$ & $\begin{array}{l}\frac{0}{0} \\
\frac{0}{2} \\
\frac{0}{Z}\end{array}$ & $\begin{array}{l}\text { FSS (Fatigue Severity Scale)* } \\
\text { CIS20-F (Checklist Individual Strength- sub-scale subjective } \\
\text { experience of fatigue)* }\end{array}$ & Cieza et al., 2005 & $b, d$ \\
\hline $\begin{array}{l}\text { Berzina et al. }{ }^{32} \\
\text { (Suíça) }\end{array}$ & & mRS (The Modified Rankin Scale)* & Cieza et al., 2005 & $b, d, e$ \\
\hline $\begin{array}{l}\text { Prodinger et al. }{ }^{69} \\
\text { (Alemanha) }\end{array}$ & & $\begin{array}{l}\text { SF-36 (Short Form Health Survey)* } \\
\text { WHODAS 2.0 (WHO Disability Schedule 2.0)* } \\
\text { SIS } 3.0 \text { (Stroke Impact Scale 3.0)*+ }\end{array}$ & $\begin{array}{l}\text { Cieza et al., 2002; } \\
\text { Cieza et al., 2005; } \\
\text { Cieza et al., } 2016\end{array}$ & $d$ \\
\hline $\begin{array}{l}\text { Sigl et al. }{ }^{67} \\
\text { (Alemanha) }\end{array}$ & & $\begin{array}{l}\text { BASFI (Bath Ankylosing Spondylitis Functional Index)* } \\
\text { DFI (Dougados Functional Index)* } \\
\text { HAQ-S (Health Assessment Questionnaire modified for the } \\
\text { spondyloarthropathies)* } \\
\text { RLDQ (Revised Leeds Disability Questionnaire)* }\end{array}$ & Cieza et al., 2002 & $b, d, e$ \\
\hline $\begin{array}{l}\text { Sigl et al. }{ }^{73} \\
\text { (Alemanha) }\end{array}$ & $\begin{array}{l}\frac{0}{0} \\
\frac{0}{0} \\
\frac{0}{0} \\
\stackrel{0}{0}\end{array}$ & $\begin{array}{l}\text { NASS (North American Spine Society Lumbar Spine Outcome } \\
\text { Assessment Instrument) } \\
\text { ODI (Oswestry Low Back Disability Questionnaire)* } \\
\text { RMDQ (Roland Morris Disability Questionnaire)* }\end{array}$ & Cieza et al., 2002 & $b, d, e$ \\
\hline $\begin{array}{l}\text { Drummond et al. }{ }^{38} \\
\text { (Brasil) }\end{array}$ & & DASH (Disabilities of the Arm, Shoulder and Hand) ${ }^{*}+$ & Cieza et al., 2005 & $b, d$ \\
\hline $\begin{array}{l}\text { Dixon et al. }{ }^{26} \\
\text { (Escócia) }\end{array}$ & & DASH (Disabilities of the Arm, Shoulder and Hand) ${ }^{*+}$ & $\begin{array}{l}\text { Nivel de concordância } \\
\text { entre avaliadores }\end{array}$ & $d$ \\
\hline
\end{tabular}


Quadro 2. Continuação

\begin{tabular}{|c|c|c|c|c|}
\hline Autor (país) & Contexto & Instrumentos que tiveram o conteúdo ligado à CIF & Procedimento de ligação & Componentes CIF \\
\hline $\begin{array}{l}\text { Alviar et al. }{ }^{60} \\
\text { (Austrália) }\end{array}$ & & $\begin{array}{l}\text { OHS (Oxford Hip Score) }{ }^{+} \\
\text {OKS (Oxford Knee Score) }{ }^{*+} \\
\text { HOOS (Hip Dysfunction and Osteoarthritis Outcome Score) }{ }^{+} \\
\text {KOOS (Knee Injury and Osteoarthritis Outcome Score) }{ }^{*} \\
\text { AIMS (Arthritis Impact Measurement Scales) }{ }^{*}\end{array}$ & Cieza et al., 2005 & $b, s, d, e$ \\
\hline $\begin{array}{l}\text { Arumugam et al. }{ }^{31} \\
\text { (Canadá) }\end{array}$ & & WLQ-26 (26-Item Work Limitations Questionnaire)* + & Cieza et al., 2005 & $b, d, e$ \\
\hline $\begin{array}{l}\text { Roe et al. }{ }^{29} \\
\text { (Noruega) }\end{array}$ & $\begin{array}{l}\frac{0}{\overline{0}} \\
\frac{0}{0} \\
\frac{0}{0} \\
\frac{0}{0}\end{array}$ & $\begin{array}{l}\text { Constant (Constant Murley shoulder Score) } \\
\text { ASES (American Shoulder and Elbow Surgeons Standardized } \\
\text { form for Assessment of the Shoulder)* } \\
\text { UCLA (University of California at Los Angeles } \\
\text { shoulder rating Scale)* } \\
\text { DASH (Disability of the Arm, Shoulder and Hand)*+ } \\
\text { SST (Simple Shoulder Test)* } \\
\text { SPADI (Shoulder Pain and Disability Index)* } \\
\text { WORC (Western Ontario Rotator Cuff Index)* } \\
\text { SRQ (Shoulder Rating Questionnaire)* } \\
\text { SDQ (Shoulder Disability Questionnaire) } \\
\text { OSS (Oxford Shoulder Score)* } \\
\text { WOSI (Western Ontario Shoulder Instability Index)* } \\
\text { QuickDASH (Shortened version of Disabilities of the Arm, } \\
\text { Shoulder and Hand)* } \\
\text { Penn (Penn shoulder score)* } \\
\text { SF-36 (36-item Short-Form Health Survey)* } \\
\text { SF-12 (12-Item Short-Form Health Survey)* } \\
\text { JCQ (Job Content Questionnaire)* } \\
\text { Nordic (Standardized Nordic questionnaires for the analysis of } \\
\text { musculoskeletal symptoms)* } \\
\text { EQ-5D (European Quality of Life Instrument-5D)* } \\
\text { FABQ (Fear-Avoidance Beliefs Questionnaire)* } \\
4 \text { DSQ (Four-Dimensional Symptom Questionnaire) }{ }^{*}\end{array}$ & Cieza et al., 2005 & $b, s, d, e$ \\
\hline $\begin{array}{l}\text { Fréz et al. }{ }^{7 \#} \\
\text { (Brasil) }\end{array}$ & & SF-36 (36-item Short-Form Health Survey)* & Consenso & $b, s, d$ \\
\hline $\begin{array}{l}\text { Forget e Higgins }{ }^{64} \\
\text { (Canadá) }\end{array}$ & & $\begin{array}{l}\text { DASH (Disabilities of the Arm, Shoulder and Hand)* } \\
\text { QuickDASH (shortened version of Disabilities of the Arm, } \\
\text { Shoulder and Hand)* } \\
\text { PRWE (Patient rated wrist evaluation) } \\
\text { PRWHE (Patient rated wrist/hand evaluation) } \\
\text { PEM (Patient Evaluation Measure) } \\
\text { MHQ (MHQ Michigan Hand Questionnaire) }{ }^{\dagger} \\
\text { UEFI (Upper extremity functional index)* }\end{array}$ & Cieza et al., 2005 & $b, d$ \\
\hline $\begin{array}{l}\text { Vincent et al. } .^{51} \\
\text { (Canadá) }\end{array}$ & & $\begin{array}{l}\text { PREE (Patient Rated Elbow Evaluation)* } \\
\text { PASES-e (American Shoulder and Elbow Surgeons Society - } \\
\text { Elbow Form)* }\end{array}$ & Cieza et al., 2005 & $b, d$ \\
\hline $\begin{array}{l}\text { Nicol et al. }{ }^{45} \\
\text { (Suiça) }\end{array}$ & & BQ (Bournemouth Questionnaire)* & Cieza et al., 2005 & $b, d$ \\
\hline $\begin{array}{l}\text { Philbois et al. }{ }^{56} \\
\text { (Brasil) }\end{array}$ & $\begin{array}{l}\frac{0}{0} \\
\frac{0}{0} \\
\frac{0}{0}\end{array}$ & $\begin{array}{l}\text { SPADI (Shoulder Pain and Disability Index)* } \\
\text { DASH (Disabilities of the Arm, Shoulder and Hand)* }\end{array}$ & $\begin{array}{l}\text { Nivel de concordância } \\
\text { entre avaliadores }\end{array}$ & $b, s, d$ \\
\hline $\begin{array}{l}\text { Fréz et al. }{ }^{65} \\
\text { (Brasil) }\end{array}$ & 气 & $\begin{array}{l}\text { AMP (Amputee Mobility Predictor)* } \\
\text { PEQ (Prosthesis Evaluation Questionnaire)* } \\
\text { HAI (Hill Assessment Index) } \\
\text { SAI (Stair Assessment Index) }\end{array}$ & Cieza et al., 2005 & e \\
\hline $\begin{array}{l}\text { De Pauw et al. }{ }^{79} \\
\text { (Bélgica) }\end{array}$ & & $\begin{array}{l}\text { TWSTRS (Toronto Western Spasmodic Torticollis Rating Scale)* } \\
\text { CDIP-58 (Cervical Dystonia Impact Profile) }{ }^{\dagger}\end{array}$ & $\begin{array}{l}\text { Cieza et al., } 2002 \\
\text { Cieza et al., } 2005\end{array}$ & $b, d$ \\
\hline $\begin{array}{l}\text { Weigl et al. }{ }^{52} \\
\text { (Alemanha) }\end{array}$ & & $\begin{array}{l}\text { WOMAC (Western Ontario and McMaster Universities)* }{ }^{*} \\
\text { LAI (Lequesne Algofunctional Index)* }\end{array}$ & Cieza et al., 2005 & $b, d, e$ \\
\hline $\begin{array}{l}\text { Stamm et al. }{ }^{75} \\
\text { (Áustria) }\end{array}$ & $\begin{array}{l}\frac{0}{\%} \\
\frac{0}{0} \\
\frac{0}{0} \\
\frac{0}{\varepsilon}\end{array}$ & $\begin{array}{l}\text { COPM (Canadian Occupational Performance Measure)* } \\
\text { AMPS (Assessment of Motor and Process Skills) } \\
\text { SODA (Sequential Occupational Dexterity Assessment) }\end{array}$ & Cieza et al., 2002 & $b, d$ \\
\hline $\begin{array}{l}\text { Stucki e Cieza } 30 \\
\text { (Alemanha) }\end{array}$ & 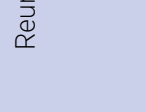 & $\begin{array}{l}\text { HAQ-DI (Health Assessment Questionnaire Disability Index)* } \\
\text { AIMS2 (Arthritis Impact Measurement Scales 2)*+ } \\
\text { SF-36 (36-item Short-Form Health Survey)* }\end{array}$ & Cieza et al., 2002 & $b, d$ \\
\hline
\end{tabular}


Quadro 2. Continuação

\begin{tabular}{|c|c|c|c|c|}
\hline Autor (país) & Contexto & Instrumentos que tiveram o conteúdo ligado à CIF & Procedimento de ligação & Componentes CIF \\
\hline $\begin{array}{l}\text { Stamm et al. }{ }^{74} \\
\text { (Áustria) }\end{array}$ & & $\begin{array}{l}\text { HAQ (Health Assessment Questionnaire)* } \\
\text { AUSCAN Index* } \\
\text { Cochin Scale (Cochin Rheumatoid Hand Disability Scale)* } \\
\text { FIHOA (Functional Index of Hand Osteoarthritis)* } \\
\text { SACRAH (Score for Assessment and Qualification of Chronic } \\
\text { Rheumatoid Affections of the Hands Questionnaire) } \\
\text { AIMS2-SF (Arthritis Impact Measurement } 2 \text { Scales short form)*+ }\end{array}$ & Cieza et al., 2005 & $b, d, e$ \\
\hline $\begin{array}{l}\text { Prodinger et al. } .^{72} \\
\text { (Áustria) }\end{array}$ & 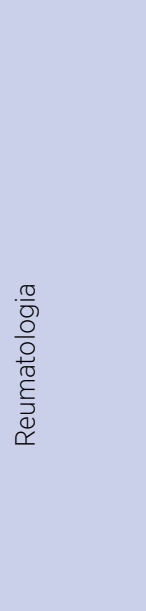 & $\begin{array}{l}\text { FIQ (Fibromyalgia Impact Questionnaire)* } \\
\text { MPQ (McGill Pain Questionnaire)* } \\
\text { SF-MPQ (Short Form McGill Pain Questionnaire)* } \\
\text { BPI (Brief Pain Inventory)* } \\
\text { LANSS (Leeds Assessment of Neuropathic Symptoms and } \\
\text { Signs Pain Scale)* } \\
\text { FACIT-FS (Functional Assessment of Chronic IIIness Therapy)*+ } \\
\text { FACIT-F (Functional Assessment of } \\
\text { Chronic Illness Therapy-Fatigue)*+ } \\
\text { FSS (Fatigue Severity Scale)* } \\
\text { MFI-20 (Multidimensional Fatigue Inventory)* } \\
\text { MAF (Multidimensional Assessment of Fatigue) } \\
\text { MOS (Sleep scale from Medical Outcomes Study) } \\
\text { SF-36 (36-item Short-Form Health Survey)* } \\
\text { ASEX (Arizona Sexual Experiences Scale)* } \\
\text { BDI (Beck Depression Inventory)* } \\
\text { HADS (Hospital Anxiety and Depression Scale)* } \\
\text { HRSD (Hamilton Rating Scale for Depression)* }\end{array}$ & Cieza et al., 2005 & $b, s, d, e$ \\
\hline $\begin{array}{l}\text { Rat et al. }{ }^{47} \\
\text { (França) }\end{array}$ & & OAKHQOL (Osteoarthritis Knee and Hip Quality of Life) & Cieza et al., 2005 & $b, d, e$ \\
\hline $\begin{array}{l}\text { Milman et al. }{ }^{66} \\
\text { (Canadá) }\end{array}$ & & $\begin{array}{l}\text { BVAS (Birmingham Vasculitis Activity Score)* } \\
\text { BVASv3 (Birmingham Vasculitis Activity Score version 3)* } \\
\text { BVAS/WG (Birmingham Vasculitis Activity Score for } \\
\text { granulomatosis with polyangiitis)* } \\
\text { VDI (Vasculitis Damege Index) } \\
\text { SF-36 (36-item Short-Form Health Survey)* }\end{array}$ & Cieza et al., 2005 & $b, s, d$ \\
\hline $\begin{array}{l}\text { Moura et al.54\# } \\
\text { (Brasil) }\end{array}$ & & SALSA (Screening of Activity Limitation \& Safety Awareness)* & Não explicitado & $d$ \\
\hline $\begin{array}{l}\text { Bladh et al. }{ }^{33} \\
\text { (Suécia) }\end{array}$ & $\begin{array}{l}\frac{0}{2} \\
\frac{0}{0} \\
\frac{0}{0} \\
\frac{0}{2}\end{array}$ & $\begin{array}{l}\text { FES-I (Falls Efficacy Scale-International) }{ }^{\dagger} \\
\text { FES(S) (Swedish version of the Falls Efficacy Scale) }{ }^{+} \\
\text {ABC (Activities-specific Balance Confidence Scale)* } \\
\text { SAFFE (modified Survey of Activities and Fear of Falling } \\
\text { in the Elderly) })^{+}\end{array}$ & Cieza et al., 2005 & $b, d$ \\
\hline $\begin{array}{l}\text { Marques et al. }{ }^{43} \\
\text { (Portugal) }\end{array}$ & $\mathbb{~}$ & EASY-Care Standard* & Cieza et al., 2005 & $b, s, d, e$ \\
\hline $\begin{array}{l}\text { Vincent et al. }{ }^{51} \\
\text { (Canadá) }\end{array}$ & & $\begin{array}{l}\text { PREE (Patient Rated Elbow Evaluation)* } \\
\text { pASES-e (American Shoulder and Elbow Surgeons Society - } \\
\text { Elbow Form)* }\end{array}$ & Cieza et al., 2005 & $b, d$ \\
\hline $\begin{array}{l}\text { Nicol et al. }{ }^{45} \\
\text { (Suiça) }\end{array}$ & & $\mathrm{BQ}$ (Bournemouth Questionnaire)* & Cieza et al., 2005 & $b, d$ \\
\hline $\begin{array}{l}\text { Philbois et al. }{ }^{56} \\
\text { (Brasil) }\end{array}$ & $\frac{\pi}{\frac{\pi}{0}}$ & $\begin{array}{l}\text { SPADI (Shoulder Pain and Disability Index)* } \\
\text { DASH (Disabilities of the Arm, Shoulder and Hand)* }\end{array}$ & $\begin{array}{l}\text { Nível de concordância } \\
\text { entre avaliadores }\end{array}$ & $b, s, d$ \\
\hline $\begin{array}{l}\text { Fréz et al. }{ }^{65} \\
\text { (Brasil) }\end{array}$ & Oे & $\begin{array}{l}\text { AMP (Amputee Mobility Predictor)* } \\
\text { PEQ (Prosthesis Evaluation Questionnaire)* } \\
\text { HAI (Hill Assessment Index) } \\
\text { SAI (Stair Assessment Index) }\end{array}$ & Cieza et al., 2005 & e \\
\hline $\begin{array}{l}\text { De Pauw et al. }{ }^{79} \\
\text { (Bélgica) }\end{array}$ & & $\begin{array}{l}\text { TWSTRS (Toronto Western Spasmodic } \\
\text { Torticollis Rating Scale)* } \\
\text { CDIP-58 (Cervical Dystonia Impact Profile) }{ }^{\dagger}\end{array}$ & $\begin{array}{l}\text { Cieza et al., } 2002 \\
\text { Cieza et al., } 2005\end{array}$ & $b, d$ \\
\hline $\begin{array}{l}\text { Weigl et al. }{ }^{52} \\
\text { (Alemanha) }\end{array}$ & $\begin{array}{l}\frac{0}{\pi} \\
\frac{0}{0}\end{array}$ & $\begin{array}{l}\text { WOMAC (Western Ontario and McMaster Universities)* } \\
\text { LAI (Lequesne Algofunctional Index)* }\end{array}$ & Cieza et al., 2005 & $b, d, e$ \\
\hline $\begin{array}{l}\text { Stamm et al. }{ }^{75} \\
\text { (Áustria) }\end{array}$ & 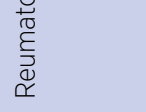 & $\begin{array}{l}\text { COPM (Canadian Occupational Performance Measure)* } \\
\text { AMPS (Assessment of Motor and Process Skills) } \\
\text { SODA (Sequential Occupational Dexterity Assessment) }\end{array}$ & Cieza et al., 2002 & $b, d$ \\
\hline
\end{tabular}


Quadro 2. Continuação

\begin{tabular}{|c|c|c|c|c|}
\hline Autor (pais) & Contexto & Instrumentos que tiveram o conteúdo ligado à CIF & Procedimento de ligação & Componentes CIF \\
\hline $\begin{array}{l}\text { Stucki e Cieza } \\
\text { (Alemanha) }\end{array}$ & & $\begin{array}{l}\text { HAQ-DI (Health Assessment Questionnaire Disability Index)* } \\
\text { AIMS2 (Arthritis Impact Measurement Scales 2)*+ } \\
\text { SF-36 (36-item Short-Form Health Survey)* }\end{array}$ & Cieza et al., 2002 & $b, d$ \\
\hline $\begin{array}{l}\text { Stamm et al. }{ }^{74} \\
\text { (Áustria) }\end{array}$ & & $\begin{array}{l}\text { HAQ (Health Assessment Questionnaire)* } \\
\text { AUSCAN Index* } \\
\text { Cochin Scale (Cochin Rheumatoid Hand Disability Scale)* } \\
\text { FIHOA (Functional Index of Hand Osteoarthritis)* } \\
\text { SACRAH (Score for Assessment and Qualification of Chronic } \\
\text { Rheumatoid Affections of the Hands Questionnaire) } \\
\text { AIMS2-SF (Arthritis Impact Measurement } 2 \text { Scales short form)*+ }\end{array}$ & Cieza et al., 2005 & $b, d, e$ \\
\hline $\begin{array}{l}\text { Prodinger et al. }{ }^{72} \\
\text { (Áustria) }\end{array}$ & 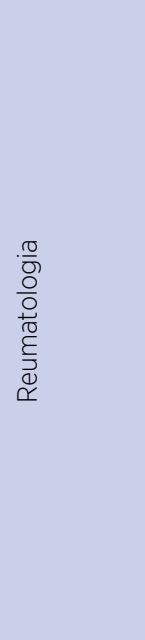 & $\begin{array}{l}\text { FIQ (Fibromyalgia Impact Questionnaire)* } \\
\text { MPQ (McGill Pain Questionnaire)* } \\
\text { SF-MPQ (Short Form McGill Pain Questionnaire)* } \\
\text { BPI (Brief Pain Inventory)* } \\
\text { LANSS (Leeds Assessment of Neuropathic Symptoms and } \\
\text { Signs Pain Scale)* } \\
\text { FACIT-FS (Functional Assessment of Chronic Illness Therapy)*+ } \\
\text { FACIT-F (Functional Assessment of Chronic Illness Therapy- } \\
\text { Fatigue)*t } \\
\text { FSS (Fatigue Severity Scale)* } \\
\text { MFI-2O (Multidimensional Fatigue Inventory)* } \\
\text { MAF (Multidimensional Assessment of Fatigue) } \\
\text { MOS (Sleep scale from Medical Outcomes Study) } \\
\text { SF-36 (36-item Short-Form Health Survey)* } \\
\text { ASEX (Arizona Sexual Experiences Scale)* } \\
\text { BDI (Beck Depression Inventory)* } \\
\text { HADS (Hospital Anxiety and Depression Scale)* } \\
\text { HRSD (Hamilton Rating Scale for Depression)* }\end{array}$ & Cieza et al., 2005 & $b, s, d, e$ \\
\hline $\begin{array}{l}\text { Rat et al. }{ }^{47} \\
\text { (França) }\end{array}$ & & OAKHQOL (Osteoarthritis Knee and Hip Quality of Life) & Cieza et al., 2005 & $b, d, e$ \\
\hline $\begin{array}{l}\text { Milman et al. }{ }^{66} \\
\text { (Canadá) }\end{array}$ & & $\begin{array}{l}\text { BVAS (Birmingham Vasculitis Activity Score)* } \\
\text { BVASv3 (Birmingham Vasculitis Activity Score version 3)* } \\
\text { BVAS/WG (Birmingham Vasculitis Activity Score for } \\
\text { granulomatosis with polyangiitis)* } \\
\text { VDI (Vasculitis Damege Index) } \\
\text { SF-36 (36-item Short-Form Health Survey)* }\end{array}$ & Cieza et al., 2005 & $b, s, d$ \\
\hline $\begin{array}{l}\text { Moura et al. } .^{54 \#} \\
\text { (Brasil) }\end{array}$ & & SALSA (Screening of Activity Limitation \& Safety Awareness)* & Não explicitado & $d$ \\
\hline $\begin{array}{l}\text { Bladh et al. }{ }^{33} \\
\text { (Suécia) }\end{array}$ & $\begin{array}{l}\frac{.0}{\circ} \\
\frac{0}{0} \\
\frac{0}{\frac{1}{0}} \\
\frac{0}{Q}\end{array}$ & $\begin{array}{l}\text { FES-I (Falls Efficacy Scale-International) }{ }^{+} \\
\text {FES(S) (Swedish version of the Falls Efficacy Scale) }{ }^{+} \\
\text {ABC (Activities-specific Balance Confidence Scale) })^{*} \\
\text { SAFFE (modified Survey of Activities and Fear of Falling in } \\
\text { the Elderly) }{ }^{+}\end{array}$ & Cieza et al., 2005 & $b, d$ \\
\hline $\begin{array}{l}\text { Marques et al. }{ }^{43} \\
\text { (Portugal) }\end{array}$ & 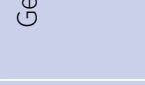 & EASY-Care Standard* & Cieza et al., 2005 & $b, s, d, e$ \\
\hline $\begin{array}{l}\text { Araujo et al. }{ }^{61 \#} \\
\text { (Brasil) }\end{array}$ & & $\begin{array}{l}\text { BI (Barthel Index)* } \\
\text { NHP (Nottingham Health Profile)* } \\
\text { MMSE (Mini-Mental State Examination)* }\end{array}$ & Consenso & $b, d$ \\
\hline $\begin{array}{l}\text { Eckert e Lange }{ }^{63} \\
\text { (Alemanha) }\end{array}$ & $\begin{array}{l}\frac{0}{\delta} \\
\frac{0}{0} \\
+\frac{1}{c} \\
\frac{0}{d} \\
\stackrel{0}{0}\end{array}$ & $\begin{array}{l}\text { 7-Day PAR (7-Day Physical Activity Recall Scale) } \\
\text { AAS (Australian Activity Survey) } \\
\text { Baecke modified (Baecke modified physical activity } \\
\text { questionnaire for the elderly)* } \\
\text { Brunel PAQ (Brunel lifestyle physical activity Questionnaire) } \\
\text { CHAMPS (Community Healthy Activities Model Program for } \\
\text { Seniors) } \\
\text { EPAQ2 (European Physical Activity Questionnaire - second } \\
\text { version) } \\
\text { EPIC-s (The European Prospective Investigation into Cancer } \\
\text { Study-Short Form) } \\
\text { GPAQ (Global Physical Activity Questionnaire)* }\end{array}$ & Cieza et al., 2005 & $d$ \\
\hline
\end{tabular}




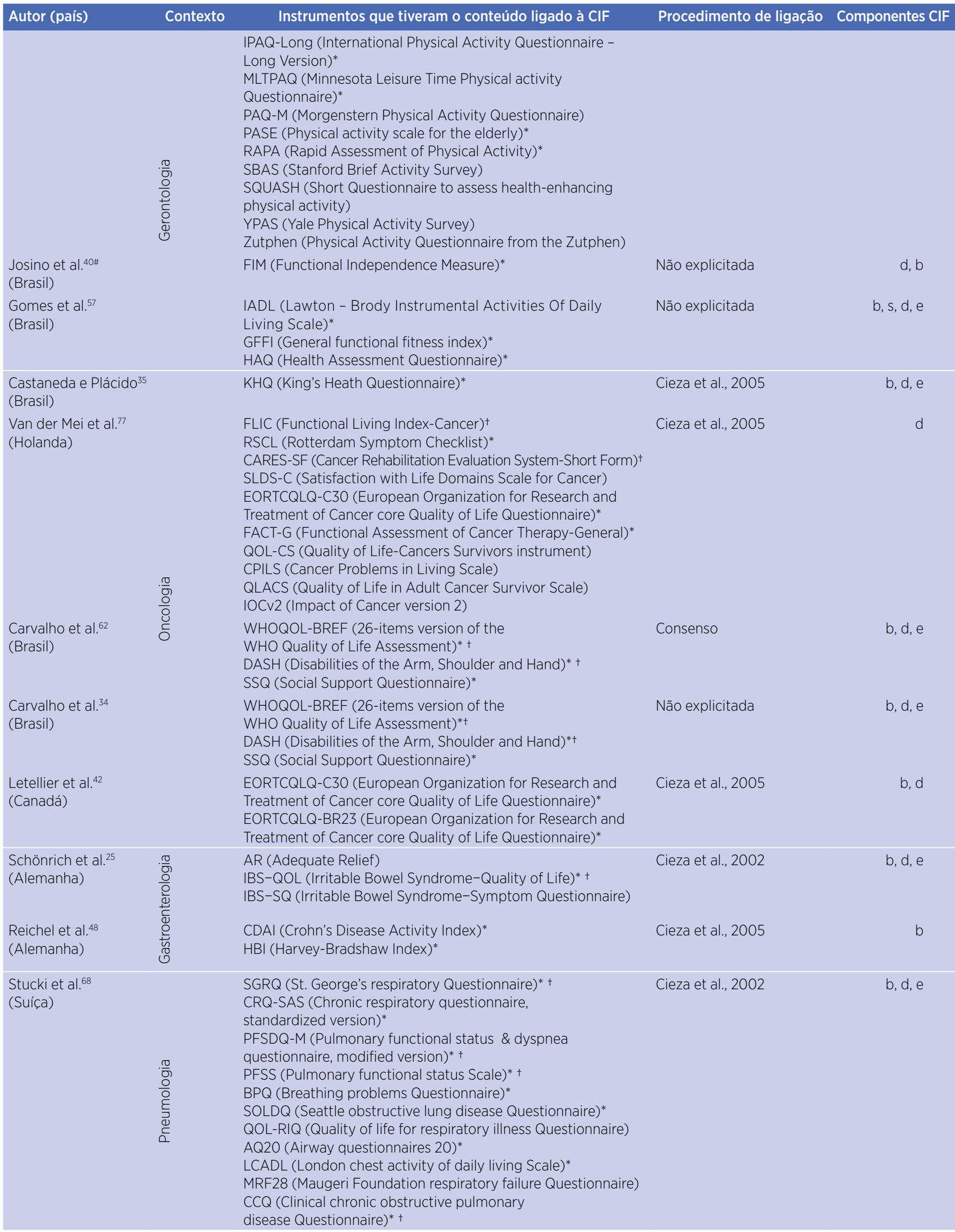


Quadro 2. Continuação

\begin{tabular}{|c|c|c|c|c|}
\hline Autor (país) & Contexto & Instrumentos que tiveram o conteúdo ligado à CIF & Procedimento de ligação & Componentes CIF \\
\hline $\begin{array}{l}\text { Raggi e Leonardi }{ }^{46} \\
\text { (Itália) }\end{array}$ & $\begin{array}{l}\text { Neuro- } \\
\text { muscular }\end{array}$ & ACTIVLIM questionnaire & Cieza et al., 2005 & $d$ \\
\hline $\begin{array}{l}\text { Zaponi et al. }{ }^{53 \#} \\
\text { (Brasil) }\end{array}$ & Cardiologia & MLHFQ (Minnesota Living with Heart Failure Questionnaire)* & Cieza et al., 2005 & $b, d, e$ \\
\hline $\begin{array}{l}\text { Dantas et al. }{ }^{70} \\
\text { (Brasil) }\end{array}$ & Urologia & $\begin{array}{l}\text { ICIQ (International Consultation on Incontinence } \\
\text { Questionnaire)* } \\
\text { KHQ (King's Health Questionnaire)* } \\
\text { IQOL (Incontinence Quality of Life Questionnaire)* } \\
\text { BSQ (Bristol Symptoms Questionnaire)* }\end{array}$ & $\begin{array}{l}\text { Cieza et al., 2002; } \\
\text { Cieza et al., 2005; } \\
\text { Cieza et al., } 2016\end{array}$ & $b, d, e, s$ \\
\hline $\begin{array}{l}\text { Cieza et al. }{ }^{10} \\
\text { (Alemanha) }\end{array}$ & & $\begin{array}{l}\text { SF-36 (36-item Short-Form Health Survey)* } \\
\text { NHP (Nottingham Health Profile)* } \\
\text { QL-I (Spitzer's Quality of Life Index)* } \\
\text { WHOQOL-BREF (26-items version of the } \\
\text { WHO Quality of Life Assessment)* } \\
\text { WHODAS II (WHO Disability Assessment Schedule)* + } \\
\text { EQ-5D (European Quality of Life Instrument - 5D)* }\end{array}$ & Cieza et al., 2002 & $b, d, e$ \\
\hline $\begin{array}{l}\text { Grill et al. }{ }^{27} \\
\text { (Alemanha) }\end{array}$ & & $\begin{array}{l}\text { FAM (Functional Assessment Measure)* } \\
\text { FIM (Functional Independence Measure)* } \\
\text { BI (Barthel index)* }\end{array}$ & Cieza et al., 2002 & $b, d, e$ \\
\hline $\begin{array}{l}\text { Guscia et al. }{ }^{23} \\
\text { (Austrália) }\end{array}$ & 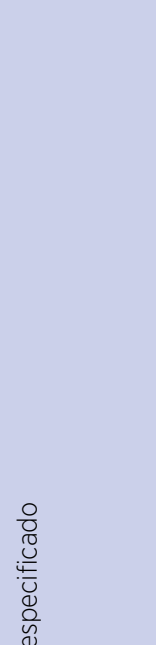 & $\begin{array}{l}\text { CDER (Client Development Evaluation Report) } \\
\text { DDP (Developmental Disability Profile) } \\
\text { DD-SNAP (Developmental Disabilities Support Needs } \\
\text { Assessment Profile) } \\
\text { Supports Intensity Scale } \\
\text { BI (Barthel Index)* } \\
\text { CHART (Craig Handicap Assessment and Reporting } \\
\text { Technique) } \\
\text { DRS (Disability Rating Scale)* } \\
\text { FIM (Functional Independence Measure)* } \\
\text { RUG-ADL (Resource Utilization Groups - ADL) }{ }^{+} \\
\text {CAN (Camberwell Assessment of Need)* } \\
\text { HoNOS (Health of the Nation Outcome Scales) }{ }^{+} \\
\text {HoNOS-65+(Health of the Nation Outcome Scales - } 65+\text { ) } \\
\text { LSP (Life Skills Profile)* } \\
\text { CANDID (Camberwell Assessment of Need for Adults with } \\
\text { Developmental and Intellectual Disabilities) } \\
\text { HoNOS-LD (Health of the Nation Outcome Scales for people } \\
\text { with Learning Disabilities) }{ }^{+}\end{array}$ & Cieza et al., 2002 & e \\
\hline $\begin{array}{l}\text { Alvarelhão et al. }{ }^{76} \\
\text { (Portugal) }\end{array}$ & $\frac{0}{2 \pi}$ & $\begin{array}{l}\text { CHEC (Community Health Environment Checklist) } \\
\text { CHIEF (Craig Hospital Inventory of Environmental Factors) } \\
\text { FABS (Facilitators and Barriers Survey) } \\
\text { HACE (Home and Community Environment Instrument) } \\
\text { NEWS (Neighborhood Environment Walkability Scale) } \\
\text { MQE (Measure of the Quality of the Environment) }\end{array}$ & Cieza et al., 2005 & $b, d, e$ \\
\hline $\begin{array}{l}\text { Darzins et al. }{ }^{37} \\
\text { (Austrália) }\end{array}$ & & $\begin{array}{l}\text { PC-PART (Personal Care Participation Assessment and } \\
\text { Resource Tool) }\end{array}$ & Cieza et al., 2005 & $d$ \\
\hline $\begin{array}{l}\text { Gao et al. } \\
\text { (China) }\end{array}$ & & $\begin{array}{l}\text { SF36 (Short Form Health Survey)* } \\
\text { WHODAS } 2.0 \text { (WHO Disability Assessment Schedule)*+ } \\
\text { WHOQOL-100 (WHO Quality of Life-100)*+ } \\
\text { BI (Barthel index)* }\end{array}$ & Cieza et al., 2005 & $b, d, e$ \\
\hline $\begin{array}{l}\text { Moura et al. }{ }^{55} \\
\text { (Brasil) }\end{array}$ & & $\begin{array}{l}\text { MDS (Model Disability Survey)* } \\
\text { IF-Br (Brazilian Functioning Index)* } \\
\text { PNS (Pesquisa Nacional em Saúde)* }\end{array}$ & Cieza et al., 2016 & $b, d, e$ \\
\hline $\begin{array}{l}\text { Scheuringer et al. }{ }^{24} \\
\text { (Alemanha) }\end{array}$ & & $\begin{array}{l}\text { SF-36 (36-item Short-Form Health Survey)* } \\
\text { SIP (Sickness Impact Profile) } \\
\text { FIM (Functional Independence Measure)* } \\
\text { BI-ADL (Barthel Index for Activities of Daily Living)* } \\
\text { mRS (The Modified Rankin Scale)* } \\
\text { BI Modified (Barthel Index Modified)*+ } \\
\text { Katz ADL (Activities of Daily Living Katz-Index) } \\
\text { FAI (Frenchay Activities Index)* } \\
\text { NEADL (Nottingham Extended Activities of Daily Living Scale) } \\
\text { RIC-FAS (Rehabilitation Institute of Chicago Functional } \\
\text { Assessment Scale) }\end{array}$ & Cieza et al., 2002 & $b, s, d$ \\
\hline
\end{tabular}


Quadro 2. Continuação

\begin{tabular}{|c|c|c|c|c|}
\hline Autor (país) & Contexto & Instrumentos que tiveram o conteúdo ligado à CIF & Procedimento de ligação & Componentes CIF \\
\hline $\begin{array}{l}\text { Scheuringer et al. }{ }^{24} \\
\text { (Alemanha) }\end{array}$ & 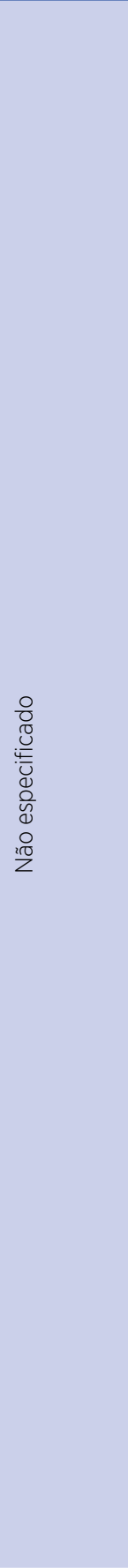 & $\begin{array}{l}\text { Karnofsky Performance Scale* } \\
\text { FAM (Functional Assessment Measure** } \\
\text { A-ADL (Australian Activities of Daily Living) } \\
\text { Lawton IADL (Lawton Instrumental Activities of Daily Living Scale)* } \\
\text { GCS (Glasgow Coma Scale)* } \\
\text { MMSE (Mini-Mental State Examination)* } \\
\text { GOS (Glasgow Outcome Scale)* } \\
\text { RLAS (Rancho Los Amigos Cognitive Functioning Scale) } \\
\text { O-log (Orientation Log) } \\
\text { KRS (Koma-Remissions-Skala) } \\
\text { Mattis DRS (Mattis Dementia Rating Scale)* } \\
\text { WMS-LMS (Wechsler Memory Scale Revised-Logical } \\
\text { Memory Subtest)* } \\
\text { WAIS-R (Wechsler Adult Intelligence Scale Revised)* } \\
\text { WMS-DSS (Wechsler Memory Scale Revised-Digit } \\
\text { Span Subtest)* } \\
\text { LOTCA (Loewenstein Occupational Therapy Cognitive } \\
\text { Assessment)* } \\
\text { GDS (Geriatric Depression Scale) } \\
\text { STAS (Spielberger's Trait Anxiety Scale)* } \\
\text { CES-D (Center for Epidemiological Studies - Depression Scale)* } \\
\text { GDS-SF (Geriatric Depression Scale - Short Form)* } \\
\text { ABS (Agitated Behaviour Scale)* } \\
\text { BDI (Beck Depression Inventory)* } \\
\text { HADS (Hospital Anxiety and Depression Scale)* } \\
\text { SDS (Zung Self-Rating Depression Scale) } \\
\text { Ashworth Spasticity Scale Modified* } \\
\text { BBS (Berg Balance Scale)* } \\
\text { Brunnstrom's States of Motor Recovery* } \\
\text { motor-FIM (Functional Independence Measure: motor score)* } \\
\text { Fugl-Meyer Motor Assessment* } \\
\text { RMI (Rivermead Mobility Index)* } \\
\text { ARAT (Action Research Armtest) } \\
\text { Buck-Gramcko-Score } \\
\text { Functional Ambulation Classification Scale* } \\
\text { AI (Ritchie Articular Index)* } \\
\text { VAS (Visual Analogue Scale for Pain)* } \\
\text { NRS (Numeric Rating Scale for Pain)* } \\
\text { BS (Braden Scale)* } \\
\text { NIHSS (National Institute of Health Stroke Scale)* } \\
\text { SSS (Scandinavian Stroke Scale)* } \\
\text { ESS (European Stroke Scale)* } \\
\text { MI (Motricity Index)* } \\
\text { MAS (Motor Assessment Scale)* } \\
\text { DRS (Disability Rating Scale)* } \\
\text { CNS (Canadian Neurological Scale)* } \\
\text { NCSE (Neurobehavioral Cognitive Status Examination) } \\
\text { BNIS (Barrow Neurological Institute Screen for Higher Cerebral } \\
\text { Function) } \\
\text { CIQ (Community Integration Questionnaire)* } \\
\text { WNSSP (Western Neuro Sensory Stimulation Profile) } \\
\text { QLMI (Quality of Life after Myocardial Infarction)* } \\
\text { RPE Borg Scale (Borg's Scale for Ratings of Perceived Exertion)* }\end{array}$ & Cieza et al., 2002 & $b, s, d$ \\
\hline
\end{tabular}

Fonte: Scharan!

Todos os instrumentos estão apresentados com nome e abreviatura em inglês, visto que nem todos têm tradução do nome para a língua portuguesa

Os países descritos no quadro referem-se ao de endereço do autor correspondente contido em cada um dos estudos, muito embora possa haver, entre os estudos da tabela, alguns que tiveram colaboração de autores de diferentes países.

Componentes da CIF: b: Funções do Corpo; s: Estruturas do Corpo; d: Atividades e Participação; e: Fatores Ambientais.

*: indica que o instrumento apresenta versão em português; t: indica que as opções de resposta do todos os itens do instrumento têm compatibilidade direta com o qualificador CIF (0, 1, 2, 3 e 4); \#: indica que o estudo realizou a classificação das categorias CIF por meio da utilização de qualificadores.

\section{DISCUSSÃo}

Os estudos identificados na presente revisão evidenciaram a utilização da CIF como referência para descrição e identificação das informações de funcionalidade coletadas por instrumentos em diferentes contextos. O número de estudos brasileiros e instrumentos com versão na língua portuguesa corroboram com Ruaro et al. ${ }^{14}$ e Castaneda and Castro $^{13}$ sobre o aumento da produção científica com a CIF no Brasil. 
A maior parte dos estudos adotou o processo metodológico de ligação, denominado de regra de ligação, proposto por Cieza et al. ${ }^{4}$ e/ou seu update por Cieza et al. ${ }^{10}$ conferindo-o como norteador para a ligação de conteúdo. $\mathrm{O}$ refinamento de tais regras ${ }^{9}$ foi utilizado por 3 estudos ${ }^{54,69,70}$. O uso desse processo metodológico não é obrigatório, o que contribui para que estudos demonstrem outras metodologias de ligação, como o consenso e o nível de concordância entre avaliadores ${ }^{61,62,78}$. Porém, a não explicitação do método de ligação limita a reprodutibilidade do estudo, o que ocorreu nos estudos de Nickel et al. ${ }^{44}$, Carvalho et al. ${ }^{34}$, Josino et al. ${ }^{40}$, Philbois et al. ${ }^{56}$, Gomes et al..$^{57}$ e Moura et al. ${ }^{54}$.

Dois estudos de revisão sobre a aplicação da CIF no Brasil identificaram Neurologia, Ortopedia e Reumatologia como os contextos mais frequentes ${ }^{12,14}$ assim como os achados do presente estudo. Isso pode estar relacionado ao fato destes contextos abordarem comumente doenças crônicas, nas quais a funcionalidade varia continuamente. Dentre os componentes da CIF o de Atividades e Participação foi o mais frequentemente ligado, denota-o como fundamental na avaliação de funcionalidade e incapacidade em diferentes contextos.

A CIF preconiza coleta de informação que abranja diferentes domínios de saúde, representados pelos seus componentes que pode ser operacionalizada pelo uso associado de diferentes instrumentos conjuntamente a outras fontes de dados, como anamnese, testes clínicos e exames complementares ${ }^{4,7,9,10,80}$ fato este demonstrado em estudos inclusos nesta revisão que para alcançar tal abrangência ligaram múltiplos instrumentos.

Quanto a exposição dos resultados de ligação, a forma mais completa, ou seja, todos os itens dos questionários e as respectivas categorias ligadas, foi observada em estudos que ligaram até quatro instrumentos. Isso não se aplicou apenas para Prodinger et al. ${ }^{69}$, que ligou blocos de questões dos 3 instrumentos ligados a blocos de categorias da CIF. Todos os estudos que ligaram menos de 20 demonstraram ao menos as categorias ou componentes da CIF por instrumento. Geyh et al. ${ }^{22}$ e Scheuringer et al. ${ }^{24}$, que ligaram um maior número de instrumentos não apresentaram de forma clara as categorias ligadas dificultando tanto a aplicação dos resultados quanto a escolha dos instrumentos mais adequados para o uso na prática clínica.

Quanto às repetições de instrumentos nos estudos, percebeu-se maior frequência para os de qualidade de vida e funcionalidade e suas utilizações em diferentes contextos entendendo que diferentes categorias podem ser selecionadas dependendo do contexto, do objetivo, do background do profissional ${ }^{9,72}$. As aparições de um mesmo instrumento em diferentes estudos denotam a relevância na literatura, inclusive na brasileira.

Seis estudos geraram classificação das categorias da CIF identificadas a partir da ligação do conteúdo dos instrumentos, esse número está em concordância com estudos que descreveram como pequena a operacionalização da codificação das categorias da CIF $^{12,14}$. Apesar desses estudos identificados terem classificado as categorias, não deixaram claro o método usado para codificá-las, tampouco se aplicaram separadamente os instrumentos e as categorias CIF ou, se geraram a codificação a partir das respostas dos instrumentos.

De acordo com Cieza et al. ${ }^{9}$, se forem compatíveis os conteúdos, as perspectivas e as opções de respostas, a princípio é viável a comparação quantitativa dos resultados de questionários com a CIF. Além disso, incentivam que pesquisadores registrem o método utilizado para codificação. Isso poderá contribuir com estudos futuros de desenvolvimento de processos metodológicos de codificação de categorias. Acredita-se que esse é um ponto determinante para operacionalizar a classificação das categorias da CIF.

Este estudo revelou que a maior parte dos instrumentos não tem opções de resposta diretamente compatíveis com os qualificadores da CIF. Frente a esse achado, é importante que estudos sejam realizados no sentido de investigar as relações psicométricas das opções de resposta dos instrumentos com os qualificadores da CIF que possam resultar em propostas de equivalência de escalas.

Os estudos selecionados apresentam limitações com relação a falta de clareza para expressar o processo metodológico de ligação utilizado, bem como os resultados da ligação. Ademais, os estudos que geraram classificação de categorias CIF também não expressaram de forma clara a metodologia usada para tal processo. Esses achados sugerem a necessidade de um maior detalhamento no método e nos resultados de estudos futuros de ligação melhorando a qualidade e a reprodutibilidade. Uma limitação do presente estudo foi a impossibilidade da busca manual de estudos primários de ligação nas referências bibliográficas contidas no material suplementar das revisões sistemáticas de Gradinger et al. ${ }^{81}$ e Fayed et al. ${ }^{5}$, as quais foram levantadas pelo processo de busca eletrônica. Tal impossibilidade, deveuse à indisponibilidade desse material mesmo após contato com os autores e compra do estudo junto à fonte de publicação. Apesar dessa consideração, não é possível 
afirmar se esses materiais enriqueceriam os achados do presente estudo, ou, se apresentariam instrumentos já contemplados na busca eletrônica.

Este estudo também identificou potencialidades para estudos futuros, como a operacionalização de categorias com opções de resposta compatíveis com a CIF,juntamente com os instrumentos da prática clínica com o intuito de explorar e descrever as propriedades psicométricas da classificação, bem como a tradução dos instrumentos com maior frequência de aparição na literatura e que não apresentavam sua versão na língua portuguesa. Além do desenvolvimento de estudos que investiguem a relação psicométrica das opções de resposta dos instrumentos com os qualificadores da CIF, como citado anteriormente.

\section{CONCLUSÃO}

A identificação dos instrumentos da prática clínica que tiveram seus conteúdos ligados à $\mathrm{CIF}$ bem como a presença da versão deles em português, pode contribuir com a aproximação da CIF à prática clínica brasileira por meio da utilização de instrumentos em língua portuguesa que já tenham o conteúdo ligado à esta classificação, permitindo além do conhecimento dos conteúdos contemplados nos instrumentos, uma possibilidade de aplicação das categorias identificadas para melhor compreensão do estado de funcionalidade dos indivíduos, fortalecendo assim, a abordagem biopsicossocial.

\section{AGRADECIMENTOS}

À Coordenação de Aperfeiçoamento de Pessoal de Nível Superior pelas bolsas de estudo concedidas às três primeiras autoras deste estudo.

\section{REFERÊNCIAS}

1. Krug E, Cieza A. Strengthening health systems to provide rehabilitation services. Bull WHO. 2017;95(3):167. doi:10.1080/ 09602011.2017 .1319391

2. World Health Organization. World Health Statistics 2016: monitoring health for the SDGs. Genève: WHO; 2016

3. Stucki G, Bickenbach J. Functioning: the third health indicator in the health system and the key indicator for rehabilitation. Eur J Phys Rehabil Med. 2017:53(1):134-8. doi:10.23736/S1973-9087.17.04565-8
4. Cieza A, Brockow T, Ewert T, Amman E, Kollerits B, Chatterji S, et al. Linking health-status measurements to the international classification of functioning, disability and health. J Rehabil Med. 2002;34(5):205-10.

5. Fayed N, Cieza A, Bickenbach JE. Linking health and healthrelated information to the ICF: a systematic review of the literature from 2001 to 2008. Disabil Rehabil. 2011;33(21-22):1941-51. doi:10.3109/09638288.2011.553704

6. Di Nubila HBV, Buchalla CM. O papel das classificações da OMS: CID e CIF nas definições de deficiência e incapacidade. Rev Bras Epidemiol. 2008;11:324-35. doi:10.1590/ S1415-790X2008000200014

7. Organização Mundial da Saúde. Como usar a CIF: um manual prático para o uso da Classificação Internacional de Funcionalidade, Incapacidade e Saúde (CIF). Versão preliminar para discussão. Versão em português. Genève: OMS; 2013 Oct [cited 2020 Nov 9]. Available from: http://www.fsp.usp.br/cbcd/ wp-content/uploads/2015/11/Manual-Pra\%CC\%81tico-da-CIF.pdf

8. Organização Mundial da Saúde. Centro Colaborador da Organização Mundial da Saúde para a Família de Classificações Internacionais em Português. CIF: Classificação Internacional de Funcionalidade, Incapacidade e Saúde. São Paulo (SP): Edusp; 2015.

9. Cieza A, Fayed N, Bickenbach J, Prodinger B. Refinements of the ICF linking rules to strengthen their potential for establishing comparability of health information. Disabil Rehabil. 2019;41(5):574-83. doi:10.3109/09638288.2016.1145258

10. Cieza A, Geyh S, Chatterji S, Kostanjsek N, Ustun B, Stucki G. ICF linking rules: an update based on lessons learned. J Rehabil Med. 2005:37(4):212-8. doi:10.1080/16501970510040263

11. Brockow T, Cieza A, Kuhlow H, Sigl T, Franke T, Harder M, et al. Identifying the concepts contained in outcome measures of clinical trials on musculoskeletal disorders and chronic widespread pain using the International Classification of Functioning, Disability and Health as a reference. J Rehabil Med. 2004;36(44):30-6. doi:10.1080/16501960410015371

12. Castaneda L, Bergmann A, Bahia L. A Classificação Internacional de Funcionalidade, Incapacidade e Saúde: uma revisão sistemática de estudos observacionais. Rev Bras Epidemiol. 2014;17(2):437-51. doi:10.1590/1809-4503201400020012

13. Castaneda L, Castro SS. Publicações brasileiras referentes à Classificação Internacional de Funcionalidade. Acta Fisiatrica. 2013;20(1):8. doi:10.5935/0104-7795.20130006

14. Ruaro JA, Ruaro MB, Souza DE, Fréz AR, Guerra RO. An overview and profile of the ICF's use in Brazil: a decade of history. Braz J Phys Ther. 2012;16:454-62. doi:10.1590/S1413-35552012005000063

15. Brasileiro IC, Moreira TMM, Buchalla CM. Classificação Internacional de Funcionalidade, Incapacidade e Saúde e seu uso no Brasil. Acta Fisistr. 2013;20(1):37-41. doi:10.5935/0104-7795.20130007

16. Moher D, Liberati A, Tetzlaff J, Altman DG. Preferred reporting items for systematic reviews and meta-analyses: the PRISMA statement. Ann Intern Med. 2009;151(4):264-9. doi:10.7326/0003-4819-151-4-200908180-00135

17. World Health Organization. World Health Assembly (WHA). Resolution WHA 54.21: International Classification of Functioning, Disability and Health. Genève: WHO; 2001[cited 2020 Nov 9]. Available from: http://www.who.int/classifications/icf/en/ 
18. Brennan P, Silman A. Statistical methods for assessing observer variability in clinical measures. BMJ. 1992;304(6840):1491-4. doi:10.1136/bmj.304.6840.1491

19. Faria CDCM, Silva SM, Corrêa JCF, Laurentino GEC, TeixeiraSalmela LF. Identificação das categorias de participação da CIF em instrumentos de qualidade de vida utilizados em indivíduos acometidos pelo acidente vascular encefálico. Rev Panam Salud Publica. 2012;31:338-44. doi:10.1590/ S1020-49892012000400011

20. Schepers V, Ketelaar M, Van de Port I, Visser-Meily J, Lindeman E. Comparing contents of functional outcome measures in stroke rehabilitation using the International Classification of Functioning, Disability and Health. Disabil Rehabil. 2007;29(3):221-30. doi:10.1080/09638280600756257

21. Geyh S, Cieza A, Kollerits B, Grimby G, Stucki G. Content comparison of health-related quality of life measures used in stroke based on the international classification of functioning, disability and health (ICF): a systematic review. Qual Life Res. 2007;16(5):833-51. doi:10.1007/s11136-007-9174-8

22. Geyh S, Kurt T, Brockow T, Cieza A, Ewert T, Omar Z, et al. Identifying the concepts contained in outcome measures of clinical trials on stroke using the International Classification of Functioning, Disability and Health as a reference. J Rehabil Med. 2004;36(44):56-62. doi:10.1080/16501960410015399

23. Guscia R, Ekberg S, Harries J, Kirby N. Measurement of environmental constructs in disability assessment instruments. JPPID. 2006;3(3):173-80. doi:10.1111/j.1741-1130.2006.00077.x

24. Scheuringer M, Grill E, Boldt C, Mittrach R, Müllner P, Stucki $G$. Systematic review of measures and their concepts used in published studies focusing on rehabilitation in the acute hospital and in early post-acute rehabilitation facilities. Disabil Rehabil. 2005;27(7-8):419-29. doi:10.1080/09638280400014089

25. Schönrich S, Brockow T, Franke T, Dembski R, Resch K, Cieza A. Analyzing the content of outcome measures in clinical trials on irritable bowel syndrome using the international classification of functioning, disability and health as a reference. Die Rehabil. 2006;45(3):172-80. doi:10.1055/s-2005-915277

26. Dixon D, Johnston M, McQueen M, Court-Brown C. The Disabilities of the Arm, Shoulder and Hand Questionnaire (DASH) can measure the impairment, activity limitations and participation restriction constructs from the International Classification of Functioning, Disability and Health (ICF). BMC Musculoskelet Disord. 2008;9:114. doi:10.1186/1471-2474-9-114

27. Grill E, Stucki G, Scheuringer M, Melvin J. Validation of International Classification of Functioning, Disability, and Health (ICF) Core Sets for early postacute rehabilitation facilities: comparisons with three other functional measures. Am J Phys Med Rehabil. 2006;85(8):640-9. doi:10.1097/01.phm.0000228537.17239.7f

28. Laxe S, Tschiesner U, Zasler N, Lopez-Blazquez R, Tormos JM, Bernabeu M. What domains of the International Classification of Functioning, Disability and Health are covered by the most commonly used measurement instruments in traumatic brain injury research? Clin Neurol Neurosurg. 2012;114(6):645-50. doi:10.1016/j.clineuro.2011.12.038

29. Roe Y, Soberg HL, Bautz-Holter E, Ostensjo S. A systematic review of measures of shoulder pain and functioning using the International classification of functioning, disability and health (ICF). BMC Musculoskelet Disord. 2013;14:73. doi:10.1186/1471-2474-14-73
30. Stucki G, Cieza A. The International Classification of Functioning, Disability and Health (ICF) core sets for rheumatoid arthritis: a way to specify functioning. Ann Rheum Dis. 2004;63(Suppl 2):ii40-ii5. doi:10.1136/ard.2004.028233

31. Arumugam V, MacDermid JC, Grewal R. Content analysis of work limitation, Stanford presenteeism, and work instability questionnaires using international classification of functioning, disability, and health and item perspective framework. Rehabil Res Pract. 2013:614825. doi:10.1155/2013/614825

32. Berzina G, Sveen U, Paanalahti M, Sunnerhagen KS. Analyzing the modified ranking scale using concepts of the international classification of functioning, disability and health. Eur J Phys Rehabil Med. 2016;52(2):203-13.

33. Bladh S, Nilsson MH, Carlsson G, Lexell J. Content analysis of 4 fear of falling rating scales by linking to the international classification of functioning, disability and health. PM\&R. 2013;5(7):573-82. doi:10.1016/j.pmrj.2013.01.006

34. Carvalho FN, Bergmann A, Koifman RJ. Functionality in women with breast cancer: the use of International Classification of Functioning, Disability and Health (ICF) in clinical practice. J Phys Ther Sci. 2014;26(5):721-30. doi:10.1589/jpts.26.721

35. Castaneda L, Plácido T. Ligação do King's Heath Questionário com a Classificação Internacional de Funcionalidade, Incapacidade e Saúde, para avaliação de pacientes com incontinência urinária pós cirurgia oncológica ginecológica. Acta Fisiatr. 2010;17(1):18-21.

36. Dahlgren A, Sand A, Larsson A, Karlsson AK, Claesson L. Linking the Klein-Bell Activities of Daily Living Scale to the International Classification of Functioning, Disability and Health. J Rehabil Med. 2013;45(4):351-7. doi:10.2340/16501977-1111

37. Darzins SW, Imms C, Di Stefano M. Measurement of activity limitations and participation restrictions: examination of ICF-linked content and scale properties of the FIM and PC-PART instruments. Disabil Rehabil. 2016;39(10):1025-38. doi:10.3109/09638288.2016.1172670

38. Drummond AS, Sampaio RF, Mancini MC, Kirkwood RN, Stamm TA. Linking the disabilities of arm, shoulder, and hand to the international classification of functioning, disability, and health. J Hand Ther. 2007;20(4):336-44. doi:10.1197/j.jht.2007.07.008

39. Fréz AR, Vignola BAP, Kaziyama HHS, Spezzano LC, Filippo TRM, Imamura M, et al. The relationship between the functional independence measure and the international classification of functioning, disability, and health core set for stroke. Acta Fisiatr. 2013;20(1):24-8. doi:10.5935/0104-7795.20130005

40. Josino JB, Costa RB, Vasconcelos TB, Domiciano BR, Brasileiro IC. Análise do estado de funcionalidade de idosos residentes em unidades de longa permanência. Rev Bras Promoç Saude. 2015;28(3):351-60. doi:10.5020/18061230.2015.p351

41. Koopman FS, Brehm MA, Heerkens YF, Nollet F, Beelen A. Measuring fatigue in polio survivors: content comparison and reliability of the Fatigue Severity Scale and the Checklist Individual Strength. J Rehabil Med. 2014;46(8):761-7. doi:10.2340/16501977-1838

42. Letellier ME, Dawes D, Mayo N. Content verification of the EORTC QLQ-C30/EORTC QLQ-BR23 with the International Classification of Functioning, Disability and Health. Qual Life Res. 2015;24(3):757-68. doi:10.1007/s11136-014-0791-8

43. Marques A, Martins A, Jácome C, Figueiredo D. Linking the EASY-Care Standard to the International Classification of Functioning, Disability and Health. Disabil Rehabil. 2014;36(7):593-9. doi:10.3109/09638288.2013.804598 
44. Nickel R, Pinto LM, Lima AP, Navarro EJ, Teive HAG, Becker N, et al. Estudo descritivo do desempenho ocupacional do sujeito com doença de Parkinson: o uso da CIF como ferramenta para classificação da atividade e participação. Acta Fisiatr. 2010;17(1):5.

45. Nicol R, Robinson Nicol M, Hopfe M, Newell D. Linking the Bournemouth Questionnaire for low back pain to the International Classification of Functioning, Disability and Health. Disabil Rehabil. 2015;38(11):1089-96. doi:10.3109/09638288.2015.1090484

46. Raggi A, Leonardi M. Assessing activity limitations in patients with neuromuscular diseases: is the ACTIVLIM questionnaire linked to ICF and ICF-CY? Int J Rehabil Res. 2009;32(2):148-53. doi:10.1097/MRR.0b013e32831e4573

47. Rat A-C, Guillemin F, Pouchot J. Mapping the osteoarthritis knee and hip quality of life (OAKHQOL) instrument to the international classification of functioning, disability and health and comparison to five health status instruments used in osteoarthritis. Rheumatology. 2008;47(11):1719-25. doi:10.1093/rheumatology/ken352

48. Reichel C, Streit J, Wunsch S. Linking Crohn's disease health status measurements with International Classification of Functioning, Disability and Health and vocational rehabilitation outcomes. J Rehabil Med. 2010;42(1):74-80. doi:10.2340/16501977-0478

49. Silva SM, Correa FI, Faria CD, Correa JC. Comparison of qualityof-life instruments for assessing the participation after stroke based on the International Classification of Functioning, Disability and Health (ICF). Braz J Phys Ther. 2013;17(5):470-8. doi:10.1590/S1413-35552012005000118

50. Teixeira-Salmela LF, Neto MG, Magalhães LC, Lima RC, Faria CD. Content comparisons of stroke-specific quality of life based upon the international classification of functioning, disability, and health. Qual Life Res. 2009;18(6):765-73. doi:10.1007/s11136-009-9488-9

51. Vincent JI, MacDermid JC, King GJ, Grewal R. Linking of the Patient Rated Elbow Evaluation (PREE) and the American Shoulder and Elbow Surgeons - Elbow questionnaire (pASES-e) to the International Classification of Functioning Disability and Health (ICF) and Hand Core Sets. J Hand Ther. 2015;28(1):61-8. doi:10.1016/j.jht.2014.10.002

52. Weigl M, Cieza A, Harder M, Geyh S, Amann E, Kostanjsek N, et al. Linking osteoarthritis-specific health-status measures to the International Classification of Functioning, Disability, and Health (ICF). Osteoarthr Cartil. 2003;11(7):519-23. doi:10.1016/ S1063-4584(03)00086-4

53. Zaponi RS, Frez AR, Mora CTR, Ruaro JA, Daniel CR. Avaliação da qualidade de vida de portadores de insuficiência cardíaca congestiva e sua correlação com a Classificação Internacional de Funcionalidade, Incapacidade e Saúde. Acta Fisiatrica. 2016;22(3):105-10. doi:10.5935/0104-7795.20150021

54. Moura EG, Araújo APM, Silva MCR, Cardoso BA, Silva Holanda MC, Conceição AO, et al. Relação entre a Classificação Internacional de Funcionalidade, Incapacidade e Saúde (CIF) ea limitação de atividades e restrição à participação de indivíduos com hanseníase. Cad Saude Colet. 2017;25(3):355-361. doi:10.1590/1414-462X201700030336

55. Moura L, Santos WR, Castro SS, Ito E, Luz e Silva DC, Yokota RTC, et al. Applying the ICF linking rules to compare populationbased data from different sources: an exemplary analysis of tools used to collect information on disability. Disabil Rehabil. 2019;41(5):601-612. doi:10.1080/09638288.2017.1370734
56. Philbois SV, Martins J, Souza CS, Sampaio RF, Oliveira AS. Health professionals identify components of the International Classification of Functioning, Disability and Health (ICF) in questionnaires for the upper limb. Braz J Phys Ther. 2016;20(1):15-25. doi:10.1590/bjpt-rbf.2014.0135

57. Gomes CS, Buranello MC, Castro SS. Assessment instruments of functioning in Brazilian elderly and the ICF: a systematic review. Fisioter Mov. 2017;30(3):625-37. doi:10.1590/ 1980-5918.030.003.AR03

58. Campos TF, Rodrigues CA, Farias I, Ribeiro TS, Melo LP. Comparação dos instrumentos de avaliação do sono, cognição e função no acidente vascular encefálico com a classificação internacional de funcionalidade, incapacidade e saúde (CIF). Braz J Phys Ther. 2012;16(1):23-9. doi:10.12707/RIII12151

59. Alghwiri AA, Marchetti GF, Whitney SL. Content comparison of self-report measures used in vestibular rehabilitation based on the international classification of functioning, disability and health. Phys Ther. 2011;91(3):346-57. doi:10.2522/ptj.20100255

60. Alviar MJ, Olver J, Brand C, Tropea J, Hale T, Pirpiris M, et al. Do patient-reported outcome measures in hip and knee arthroplasty rehabilitation have robust measurement attributes? A systematic review. J Rehabil Med. 2011;43(7):572-83. doi:10.2340/16501977-0828

61. Araujo LB, Moreira NB, Villegas ILP, Loureiro APC, Israel VL, Gato SA, et al. Investigação dos saberes quanto à capacidade funcional e qualidade de vida em idosas institucionalizadas, sob a ótica da CIF. Acta Fisistr. 2015;22(3):1117. doi:10.5935/0104-7795.20150022

62. Carvalho FN, Koifman RJ, Bergmann A. International Classification of Functioning, Disability, and Health in women with breast cancer: a proposal for measurement instruments. Cad Saude Publica. 2013;29:1083-93. doi:10.1590/ S0102-311X2013000600005

63. Eckert KG, Lange MA. Comparison of physical activity questionnaires for the elderly with the International Classification of Functioning, Disability and Health (ICF): an analysis of content. BMC Public Health. 2015;15:249. doi:10.1186/ s12889-015-1562-3

64. Forget NJ, Higgins J. Comparison of generic patient-reported outcome measures used with upper extremity musculoskeletal disorders: linking process using the International Classification of Functioning, Disability, and Health (ICF). J Rehabil Med. 2014;46(4):327-34. doi:10.2340/16501977-1784

65. Fréz AR, Binda AC, Dubiela A, Daniel CR, Bertolini GRF, Ruaro $J A$, et al. Functional profile of active older adults with low back pain, according to the ICF. Rev Bras Med Esporte. 2016;22:252-5. doi:10.1590/1517-869220162204159647

66. Milman N, Boonen A, Merkel PA, Tugwell P. Mapping of the outcome measures in rheumatology core set for antineutrophil cytoplasmic antibody-associated vasculitis to the International Classification of Function, Disability and Health. Arthritis Care Res. 2015;67(2):255-63. doi:10.1002/acr.22414

67. Sigl T, Cieza A, van der Heijde D, Stucki G. ICF based comparison of disease specific instruments measuring physical functional ability in ankylosing spondylitis. Ann Rheum Dis. 2005;64(11):1576-81. doi:10.1136/ard.2004.027185

68. Stucki A, Stucki G, Cieza A, Schuurmans MM, Kostanjsek $\mathrm{N}$, Ruof J. Content comparison of health-related quality of 
life instruments for COPD. Respir Med. 2007;101(6):1113-22. doi:10.1016/j.rmed.2006.11.016

69. Prodinger B, Tennant A, Stucki G. Standardized reporting of functioning information on ICF-based common metrics. Eur J Phys Rehabil Med. 2018;54(1):110-7. doi:10.23736/S1973-9087.17.04784-0

70. Dantas THM, Castaneda L, Magalhães AG, Dantas DS. Linking of assessment scales for women with urinary incontinence and the International Classification of Functioning, Disability and Health. Disabil Rehabil. 2018;29:1-7. doi:10.1080/09638288.2018.1431695.

71. Gao Y, Yan T, You L, Li K. Developing operational items for the International Classification of Functioning, Disability and Health Rehabilitation Set: the experience from China. Int J Rehabil Res. 2018;41(1):20-27. doi:10.1097/MRR.0000000000000254

72. Prodinger B, Cieza A, Williams DA, Mease P, Boonen A, KerschanSchind $\mathrm{K}$, et al. Measuring health in patients with fibromyalgia: content comparison of questionnaires based on the International Classification of Functioning, Disability and Health. Arthritis Rheum. 2008;59(5):650-8. doi:10.1002/art.23559

73. Sigl T, Cieza A, Brockow T, Chatterji S, Kostanjsek N, Stucki G. Content comparison of low back pain-specific measures based on the International Classification of Functioning, Disability and Health (ICF). Clin J Pain. 2006;22(2):147-53. doi:10.1097/01.ajp.0000155344.22064.f4

74. Stamm T, Geyh S, Cieza A, Machold K, Kollerits B, Kloppenburg $M$, et al. Measuring functioning in patients with hand osteoarthritis: content comparison of questionnaires based on the International Classification of Functioning, Disability and Health (ICF). Rheumatology. 2006;45(12):1534-41. doi:10.1093/rheumatology/kel133

75. Stamm TA, Cieza A, Machold KP, Smolen JS, Stucki G. Content comparison of occupation-based instruments in adult rheumatology and musculoskeletal rehabilitation based on the International Classification of Functioning, Disability and Health. Arthritis Rheum. 2004;51(6):917-24. doi:10.1002/art.20842

76. Alvarelhão J, Silva A, Martins A, Queiros A, Amaro A, Rocha $\mathrm{N}$, et al. Comparing the content of instruments assessing environmental factors using the International Classification of Functioning, Disability and Health. J Rehabil Med. 2012;44(1):1-6. doi:10.2340/16501977-0905

77. Van der Mei SF, Dijkers MPJM, Heerkens YF. Participation as an outcome measure in psychosocial oncology: content of cancer-specific health-related quality of life instruments. Qual Life Res. 2011;20(10):1617-27. doi:10.1007/s11136-011-9900-0

78. Fréz AR, Abdallah AA, Riedi C, Galindo J, Ruaro JA, Ribeiro SC. Proposed use of the international classification of functioning, disability and health to evaluate quality of life after an amputation. Fisioter Mov. 2014;27(1):49-56. doi:10.1590/0103-5150.027.001.A005

79. De Pauw J, van der Velden K, Cox R, Truijen S, Cras P, Mercelis R, et al. Measuring disability in patients with cervical dystonia according to the international classification of functioning, disability and health. OTJR. 2017;37(3):132-40. doi:10.1177/1539449217697043

80. Cieza A, Stucki G. Content comparison of health-related quality of life (HRQOL) instruments based on the international classification of functioning, disability and health (ICF). Qual Life Res. 2005;14(5):1225-37. doi:10.1007/s11136-007-9174-8

81. Gradinger F, Glassel A, Bentley A, Stucki A. Content comparison of 115 health status measures in sleep medicine using the International Classification of Functioning, Disability and Health (ICF) as a reference. Sleep Med Rev. 2011;15(1):33-40. doi:10.1016/j.smrv.2010.07.001 